NASA/CR-1998-206903

ICASE Report No. 98-2

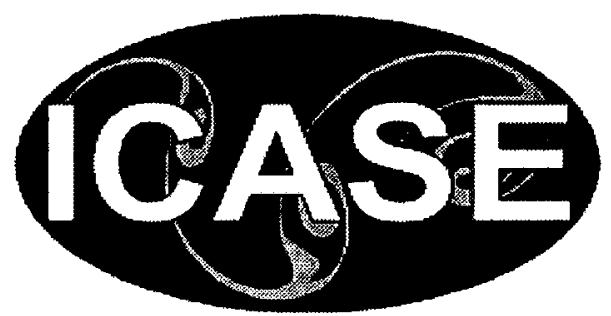

\title{
A Discontinuous Galerkin Finite Element Method for Hamilton-Jacobi Equations
}

Changqing $\mathrm{Hu}$

Brown University

Chi-Wang Shu

Brown University

Institute for Computer Applications in Science and Engineering NASA Langley Research Center

Hampton, VA

Operated by Universities Space Research Association

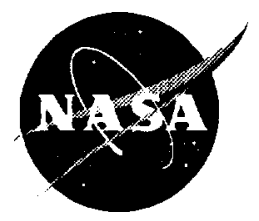

National Aeronautics and

Space Administration

Langley Research Center

Hampton, Virginia 23681-2199 
Available from the following:

NASA Center for AeroSpace Information (CASI) 800 Elkridge Landing Road

Linthicum Heights, MD 21090-2934

(301) 621-0390
National Technical Information Service (NTIS)

5285 Port Royal Road

Springfield, VA 22161-2171

(703) 487-4650 


\title{
A DISCONTINUOUS GALERKIN FINITE ELEMENT METHOD FOR HAMILTON-JACOBI EQUATIONS
}

\author{
CHANGQING HU AND CHI-WANG SHU *
}

\begin{abstract}
In this paper, we present a discontinuous Galerkin finite element method for solving the nonlinear Hamilton-Jacobi equations. This method is based on the Runge-Kutta discontinuous Galerkin finite element method for solving conservation laws. The method has the flexibility of treating complicated geometry by using arbitrary triangulation, can achieve high order accuracy with a local, compact stencil, and are suited for efficient parallel implementation. One and two dimensional numerical examples are given to illustrate the capability of the method.
\end{abstract}

Key words. Hamilton-Jacobi Equations, discontinuous Galerkin, high order accuracy.

Subject classification. Applied and Numerical Mathematics

1. Introduction. In this paper we consider the numerical solutions of the Hamilton-Jacobi (HJ) equations

$$
\phi_{t}+H\left(\phi_{x_{1}}, \ldots, \phi_{x_{d}}\right)=0, \quad \phi(x, 0)=\phi^{0}(x) .
$$

As is well known, the solutions to (1.1) are Lipschitz continuous but may have discontinuous derivatives, regardless of the smoothness of the initial condition $\phi^{0}(x)$. The non-uniqueness of such solutions also necessitates the definition of viscosity solutions, to single out a unique, practically relevant solution. (See Crandall and Lions [11]).

A widely applied class of numerical schemes for (1.1) is the class of finite difference schemes. In [12], Crandall and Lions proved the convergence of monotone finite difference schemes to the viscosity solutions of (1.1). Unfortunately, monotone schemes are at most first order accurate, measured by local truncation errors in smooth regions of the solution. In Osher and Sethian [22] and Osher and Shu [23], a class of high order essentially non-oscillatory (ENO) schemes were introduced, which were adapted from the methodologies for hyperbolic conservation laws ([14], [25], [26]). The numerical results in [22] and [23] indicate convergence to the viscosity solutions of (1.1) with high order accuracy in smooth regions, and with sharp resolution of the discontinuous derivatives. Recently, weighted ENO (WENO) schemes for conservation laws ([20], [17]) have also been adapted to the Hamilton-Jacobi equations (1.1), ([15]).

Finite difference methods require a structured mesh, hence are difficult to apply to complicated geometry or for adaptive mesh refinements. Finite volume schemes, which are based on arbitrary triangulation, are thus attractive in such cases. First order monotone type finite volume schemes were studied in [2]. A second order ENO type finite volume scheme is developed in [19]. However, higher order finite volume schemes face the problem of reconstruction on arbitrary triangulation, which is quite complicated. (See, e.g., [1]).

The Runge-Kutta discontinuous Galerkin method $([5,6,7,8,9])$ is a method devised to numerically solve the conservation law (CL):

$$
u_{t}+f_{1}(u)_{x_{1}}+\cdots+f_{d}(u)_{x_{d}}=0, \quad u(x, 0)=u^{0}(x)
$$

* Division of Applied Mathematics, Brown University, Providence, RI 02912 (e-mail: hu@cfm.brown.edu, shu@cfm.brown.edu). Research of the second author was partially supported by ARO grant DAAG55-97-1-0318, NSF grants DMS-9500814, ECS-9627849 and INT-9601084, NASA Langley grant NAG-1-1145 and Contract NAS1-19480 while in residence at ICASE, NASA Langley Research Center, Hampton, VA 23681-0001, and AFOSR grant F49620-96-1-0150. 
The method has the following attractive properties:

- It can be designed for any order of accuracy in space and time. In fact, $p$-version or spectral element type version can be designed ([21]);

- It is an explicit method, thus efficient for solving the hyperbolic problem (1.2). No global linear or nonlinear systems must be solved;

- It combines the flexibility of finite element methods in the easy handling of complicated geometry, with the high resolution property for discontinuous solutions of finite difference and finite volume methods through monotone fluxes or approximate Riemann solvers applied at the element interfaces and limiters;

- It has nice stability properties: a local cell entropy inequality for the square entropy can be proven ([16]) for general triangulation for any scalar nonlinear conservation laws (1.2) in any spatial dimensions and for any order of accuracy, without the need of nonlinear limiters. This implies nonlinear $L^{2}$ stability and entropy consistency even for discontinuous solutions;

- The method is highly compact: the evolution of the information in any element depends only on the information of itself and its immediate neighbors, regardless of the order of accuracy. This is in contrast with high order finite volume schemes which must use wide stencils for the high order reconstruction. This compactness is responsible for the efficient parallel implementation of the method, see, e.g. [3].

For details of the Runge-Kutta discontinuous Galerkin method, see the references listed above and also the review paper of Cockburn [4]. This method can also be generalized to solve problems containing higher derivatives ([10]), which is important for some of our applications in Sect. 4.

It is well known that the Hamilton-Jacobi equation (1.1) is closely related to the conservation law (1.2), in fact in one space dimension $d=1$ they are equivalent if one takes $\phi=u_{x}$. It is thus not surprising that many successful numerical methods for the Hamilton-Jacobi equation (1.1) are adapted from those for the conservation law (1.2). The high order finite difference ENO methods in [22] and [23] are such examples.

In this paper we adapt the Runge-Kutta discontinuous Galerkin method to solve the Hamilton-Jacobi equation (1.1). In Sect. 2 the algorithm in one space dimension is described and discussed. In Sect. 3 the algorithm for two space dimensions is developed. Numerical examples of one and two spatial dimensions are presented in Sect. 4. Concluding remarks are given in Sect. 5.

2. One Dimensional Case. In one space dimension (1.1) becomes

$$
\phi_{t}+H\left(\phi_{x}\right)=0, \quad \phi(x, 0)=\phi^{0}(x) .
$$

This is a relatively easy case because (2.1) is equivalent to the conservation law

$$
u_{t}+H(u)_{x}=0, \quad u(x, 0)=u^{0}(x)
$$

if we identify $u=\phi_{x}$.

Assuming (2.1) is solved in the interval $a \leq x \leq b$ and it is divided into the following cells:

$$
a=x_{\frac{1}{2}}<x_{\frac{3}{2}}<\cdots<x_{N+\frac{1}{2}}=b
$$

we denote

$$
I_{j}=\left(x_{j-\frac{1}{2}}, x_{j+\frac{1}{2}}\right), x_{j}=\frac{1}{2}\left(x_{j-\frac{1}{2}}+x_{j+\frac{1}{2}}\right), h_{j}=x_{j+\frac{1}{2}}-x_{j-\frac{1}{2}}, h=\max _{j} h_{j}
$$


and define the following approximation space

$$
V_{h}^{k}=\left\{v:\left.v\right|_{I_{j}} \in P^{k}\left(I_{j}\right), j=1, \ldots, N\right\} .
$$

Here $P^{k}\left(I_{j}\right)$ is the set of all polynomials of degree at most $k$ on the cell $I_{j}$.

A $k$-th order discontinuous Galerkin scheme for the one dimensional Hamilton-Jacobi equation (2.1) can then be defined as follows: find $\varphi \in V_{h}^{k}$, such that

$$
\int_{I_{j}} \varphi_{x t} v d x-\int_{I_{j}} H\left(\varphi_{x}\right) v_{x} d x+\hat{H}_{j+\frac{1}{2}} v_{j+\frac{1}{2}}^{-}-\hat{H}_{j-\frac{1}{2}} v_{j-\frac{1}{2}}^{+}=0, \quad j=1, \ldots, N
$$

for all $v \in V_{h}^{k-1}$. Here

$$
\hat{H}_{j+\frac{1}{2}}=\hat{H}\left(\left(\varphi_{x}\right)_{j+\frac{1}{2}}^{-},\left(\varphi_{x}\right)_{j+\frac{1}{2}}^{+}\right)
$$

is a monotone flux, i.e. $\hat{H}$ is non-decreasing in the first argument and non-increasing in the second, symbolically $\hat{H}(\uparrow, \downarrow)$, is Lipschitz continuous in both arguments, and is consistent, i.e. $\hat{H}(u, u)=H(u)$. We will mainly use the simple (local) Lax-Friedrichs flux

$$
\hat{H}\left(u^{-}, u^{+}\right)=H\left(\frac{u^{-}+u^{+}}{2}\right)-\frac{1}{2} \alpha\left(u^{+}-u^{-}\right)
$$

where $\alpha=\max _{u}\left|H^{\prime}(u)\right|$ with the maximum taken over the range covered by $u^{-}$and $u^{+}$. For other monotone fluxes, e.g. the Godunov flux (see [23]). Notice that the method described above is exactly the discontinuous Galerkin method for the conservation law equation (2.2) satisfied by the derivative $u=\phi_{x}$. This only determines $\varphi$ for each element up to a constant, since it is only a scheme for $\varphi_{x}$. The missing constant can be obtained in one of the following two ways:

1. By requiring that

$$
\int_{I_{j}}\left(\varphi_{t}+H\left(\varphi_{x}\right)\right) v d x=0, \quad j=1, \ldots, N
$$

for all $v \in V_{h}^{0}$, that is,

$$
\int_{I_{j}}\left(\varphi_{t}+H\left(\varphi_{x}\right)\right) d x=0, \quad j=1, \ldots, N
$$

2. By using (2.10) to update only one (or a few) elements, e.g., the left-most element $I_{1}$, then use

$$
\varphi\left(x_{j}, t\right)=\varphi\left(x_{1}, t\right)+\int_{x_{1}}^{x_{j}} \varphi_{x}(x, t) d x
$$

to determine the missing constant for the cell $I_{j}$.

Both approaches are used in our numerical experiments. They perform similarly for smooth problems, with the first approach giving slightly better results. However, it is our numerical experience that, when there are singularities in the derivatives, the first approach will often produce dents and bumps when the integral path in time passes through the singularities at some earlier time. The philosophy of using the second approach is that one could update only a few elements whose time integral paths do not cross derivative singularities. The numerical results shown in Sect. 4 are obtained with the second approach.

About the stability of the method proposed above, we can quote the following result of Jiang and Shu [16]. Here we assume compact support or periodic boundary condition for $\varphi$. 
Lemma 2.1. [16]. The following $L^{2}$ stability result for the derivative $\varphi_{x}$ holds for the discontinuous Galerkin method defined above, of any order of accuracy $k$ applied to any nonlinear Hamilton-Jacobi equation (2.1):

$$
\frac{d}{d t} \int_{a}^{b} \varphi_{x}^{2} d x \leq 0
$$

For a finite interval $[a, b],(2.12)$ trivially implies TVB (total variation bounded) property for the numerical solution $\varphi$ :

$$
T V(\varphi)=\int_{a}^{b}\left|\varphi_{x}\right| d x \leq \sqrt{b-a} \sqrt{\int_{a}^{b}\left(\frac{d}{d x} \phi^{0}(x)\right)^{2} d x}
$$

This is a rather strong stability result, considering that it applies even if the derivative of the solution $\phi_{x}$ develops discontinuities, no limiter has been added to the numerical scheme, and the scheme can be of arbitrary high order in accuracy. It also implies convergence of at least a subsequence of the numerical solution $\varphi$ when $h \rightarrow 0$. However, this stability result is not strong enough to imply that the limit solution is the viscosity solution of $(2.1)$.

Up to now we have only described the spatial discretization and have left the time variable $t$ continuous (the method of lines approach). Time discretization is by the TVD (total variation diminishing) high order Runge-Kutta methods developed in [25] (see also [13]). The second and third order versions used in this paper are as follows: for solving the method of lines ODE

$$
\varphi_{t}=L(\varphi)
$$

the second order TVD Runge-Kutta method is given by

$$
\begin{aligned}
\varphi^{(1)} & =\varphi^{n}+\Delta t L\left(\varphi^{n}\right) \\
\varphi^{n+1} & =\frac{1}{2} \varphi^{n}+\frac{1}{2} \varphi^{(1)}+\frac{1}{2} \Delta t L\left(\varphi^{(1)}\right),
\end{aligned}
$$

and the third order TVD Runge-Kutta method is given by

$$
\begin{aligned}
\varphi^{(1)} & =\varphi^{n}+\Delta t L\left(\varphi^{n}\right) \\
\varphi^{(2)} & =\frac{3}{4} \varphi^{n}+\frac{1}{4} \varphi^{(1)}+\frac{1}{4} \Delta t L\left(\varphi^{(1)}\right) \\
\varphi^{n+1} & =\frac{1}{3} \varphi^{n}+\frac{2}{3} \varphi^{(2)}+\frac{2}{3} \Delta t L\left(\varphi^{(2)}\right) .
\end{aligned}
$$

These Runge-Kutta methods will also be used for the two dimensional case discussed in the next section.

3. Two Dimensional Case. We consider in this section the case of two spatial dimensions. The algorithm in more spatial dimensions is similar. This time, the scalar Hamilton-Jacobi equation

$$
\phi_{t}+H\left(\phi_{x}, \phi_{y}\right)=0, \quad \phi(x, y, 0)=\phi^{0}(x, y)
$$

is in some sense equivalent to the following conservation law system

$$
u_{t}+H(u, v)_{x}=0, \quad v_{t}+H(u, v)_{y}=0, \quad(u, v)(x, y, 0)=(u, v)^{0}(x, y) .
$$

if we identify

$$
(u, v)=\left(\phi_{x}, \phi_{y}\right)
$$


For example, a vanishing viscosity solution of (3.1) corresponds, via (3.3), to a vanishing viscosity solution of (3.2), and vice versa ([18]). However, (3.2) is not a strictly hyperbolic system, which may cause problems in its numerical solution if we treat $u$ and $v$ as independent variables. Instead, we would like to still use $\phi$ as our solution variable (a polynomial) and take its derivatives as $u$ and $v$.

Assuming we are solving (3.1) in the domain $\Omega$, which has a triangulation $\mathcal{T}_{h}$ consisting of triangles or general polygons of maximum size (diameter) $h$, with the following approximation space

$$
V_{h}^{k}=\left\{v:\left.v\right|_{K} \in P^{k}(K), \forall K \in \mathcal{T}_{h}\right\},
$$

where $P^{k}(K)$ is again the set of all polynomials of degree at most $k$ on the cell $K$. We propose a discontinuous Galerkin method for (3.1) as follows: find $\varphi \in V_{h}^{k}$, such that

$$
\int_{K} \varphi_{x t} v d x d y-\int_{K} H\left(\varphi_{x}, \varphi_{y}\right) v_{x} d x d y+\sum_{e \in \partial K} \int_{e} \hat{H}_{1, e, K} v d \Gamma=0
$$

and

$$
\int_{K} \varphi_{y t} v d x d y-\int_{K} H\left(\varphi_{x}, \varphi_{y}\right) v_{y} d x d y+\sum_{e \in \partial K} \int_{e} \hat{H}_{2, e, K} v d \Gamma=0
$$

for all $v \in V_{h}^{k-1}$ and all $K \in \mathcal{T}_{h}$, in a least square sense. Here the numerical flux is

$$
\hat{H}_{i, e, K}=\hat{H}_{i, e, K}\left((\nabla \varphi)^{i n t(K)},(\nabla \varphi)^{e x t(K)}\right), \quad i=1,2
$$

where the superscript $\operatorname{int}(K)$ implies that the value is taken from within the element $K$, and the superscript $\operatorname{ext}(K)$ implies that the value is taken from outside the element $K$ and within the neighboring element $K^{\prime}$ sharing the edge $e$ with $K$. The flux (3.7) satisfies the following properties:

1. $\hat{H}_{i, e, K}$ is Lipschitz continuous with respect to all its arguments;

2. Consistency:

$$
\hat{H}_{i, e, K}(\nabla \phi, \nabla \phi)=H(\nabla \phi) n_{i}
$$

where $n=\left(n_{1}, n_{2}\right)$ is the unit outward normal to the edge $e$ of the element $K$;

3. Conservation:

$$
\hat{H}_{i, e, K}\left((\nabla \varphi)^{i n t(K)},(\nabla \varphi)^{\operatorname{ext}(K)}\right)=-\hat{H}_{i, e, K^{\prime}}\left((\nabla \varphi)^{i n t\left(K^{\prime}\right)},(\nabla \varphi)^{\operatorname{ext}\left(K^{\prime}\right)}\right) .
$$

where $K \cap K^{\prime}=e$.

We will again mainly use the simple (local) Lax-Friedrichs flux

$$
\hat{H}_{1, e, K}\left((u, v)^{-},(u, v)^{+}\right)=H\left(\frac{u^{-}+u^{+}}{2}, \frac{v^{-}+v^{+}}{2}\right) n_{1}-\frac{1}{2} \alpha\left(u^{+}-u^{-}\right)
$$

and

$$
\hat{H}_{2, e, K}\left((u, v)^{-},(u, v)^{+}\right)=H\left(\frac{u^{-}+u^{+}}{2}, \frac{v^{-}+v^{+}}{2}\right) n_{2}-\frac{1}{2} \beta\left(v^{+}-v^{-}\right)
$$

where $\alpha=\max _{u, v}\left|\frac{\partial H(u, v)}{\partial u}\right|$ and $\beta=\max _{u, v}\left|\frac{\partial H(u, v)}{\partial v}\right|$, with the maximum being taken over the relevant (local) range.

Notice that (3.5)-(3.6) are exactly the discontinuous Galerkin method for the conservation law system (3.2) satisfied by the derivatives $(u, v)=\nabla \phi$. For a rectangular mesh and for $k=1$ this recovers the (local) Lax-Friedrichs monotone scheme ([23]) for (3.1) if we identify $u_{i j}=\frac{\phi_{i+1, j}-\phi_{i-1, j}}{2 \Delta x}$ and $v_{i j}=\frac{\phi_{i, j+1}-\phi_{i, j-1}}{2 \Delta y}$. 
Of course, (3.5)-(3.6) have more equations than the number of degrees of freedoms (an over-determined system) for $k>1$, thus a least square solution is needed. In practice, the least square procedure is performed as follows: we first evolve (3.2) for one time step (one inner stage for high order Runge-Kutta methods), using the discontinuous Galerkin method (3.5)-(3.6) with $u=\varphi_{x}$ and $v=\varphi_{y}$; then $\varphi$ at the next time level (stage) is obtained (up to a constant) by least square:

$$
\left\|\left(\varphi_{x}-u\right)^{2}+\left(\varphi_{y}-v\right)^{2}\right\|_{L^{1}(K)}=\min _{\psi \in P^{k}(K)}\left\|\left(\psi_{x}-u\right)^{2}+\left(\psi_{y}-v\right)^{2}\right\|_{L^{1}(K)}
$$

This determines $\varphi$ for each element up to a constant, since it is only a scheme for $\nabla \varphi$. The missing constant can again be obtained in one of the following two ways:

1. By requiring that

$$
\int_{K}\left(\varphi_{t}+H\left(\varphi_{x}, \varphi_{y}\right)\right) v d x d y=0
$$

for all $v \in V_{h}^{0}$ and for all $K \in \mathcal{T}_{h}$, that is,

$$
\int_{K}\left(\varphi_{t}+H\left(\varphi_{x}, \varphi_{y}\right)\right) d x d y=0, \quad \forall K \in \mathcal{T}_{h}
$$

2. By using (3.11) to update only one (or a few) elements, e.g., the corner element(s), then use

$$
\varphi(B, t)=\varphi(A, t)+\int_{A}^{B}\left(\varphi_{x} d x+\varphi_{y} d y\right)
$$

to determine the missing constant. The path should be taken to avoid crossing a derivative discontinuity, if possible.

In Sect. 4 we will only show numerical results obtained with the second approach, see the remarks in the previous section for the one dimensional case.

We remark that the procedure discussed above is easily implemented in any triangulation, e.g., for both rectangles and triangles.

4. Numerical Examples. Example 4.1. One dimensional Burgers' equation:

$$
\left\{\begin{array}{l}
\phi_{t}+\frac{\left(\phi_{x}+1\right)^{2}}{2}=0, \quad-1<x<1 \\
\phi(x, 0)=-\cos (\pi x)
\end{array}\right.
$$

with periodic boundary conditions.

The local Lax-Friedrichs flux (2.8) is used. At $t=0.5 / \pi^{2}$, the solution is still smooth. We list the errors and the numerical orders of accuracy in Table 4.1. We observe that, except for the $P^{1}$ case which seems to be only first order, $P^{k}$ for $k>1$ seems to provide close to $(k+1)$-th order accuracy.

At $t=3.5 / \pi^{2}$, the solution has developed a discontinuous derivative. In Fig. 4.1, we show the sharp corner-like numerical solution with 41 elements obtained with $P^{k}$ for $k=1,2,3,4$. Here and below, the solid line is the exact solution, the circles are numerical solutions (only one point per element is drawn).

Example 4.2. One dimensional equation with a non-convex flux:

$$
\left\{\begin{array}{l}
\phi_{t}-\cos \left(\phi_{x}+1\right)=0, \quad-1<x<1 \\
\phi(x, 0)=-\cos (\pi x)
\end{array}\right.
$$

with periodic boundary conditions. 
TABLE 4.1

Accuracy for $1 D$ Burgers equation, $t=0.5 / \pi^{2}$.

\begin{tabular}{|r|r|r|r|r|r|r|r|r|}
\hline & \multicolumn{2}{|c|}{$P^{1}$} & \multicolumn{2}{c|}{$P^{2}$} & \multicolumn{2}{c|}{$P^{3}$} & \multicolumn{2}{c|}{$P^{4}$} \\
\hline$N$ & $L^{1}$ error & order & $L^{1}$ error & order & $L^{1}$ error & order & $L^{1}$ error & order \\
\hline 10 & $0.17 \mathrm{E}+00$ & - & $0.14 \mathrm{E}-02$ & - & $0.21 \mathrm{E}-03$ & $-\cdots$ & $0.57 \mathrm{E}-05$ & - \\
\hline 20 & $0.78 \mathrm{E}-01$ & 1.12 & $0.18 \mathrm{E}-03$ & 2.92 & $0.13 \mathrm{E}-04$ & 3.94 & $0.73 \mathrm{E}-06$ & 2.97 \\
\hline 40 & $0.35 \mathrm{E}-01$ & 1.16 & $0.24 \mathrm{E}-04$ & 2.97 & $0.75 \mathrm{E}-06$ & 4.17 & $0.32 \mathrm{E}-07$ & 4.52 \\
\hline 80 & $0.16 \mathrm{E}-01$ & 1.12 & $0.28 \mathrm{E}-05$ & 3.08 & $0.43 \mathrm{E}-07$ & 4.12 & $0.12 \mathrm{E}-08$ & 4.79 \\
\hline 160 & $0.76 \mathrm{E}-02$ & 1.02 & $0.31 \mathrm{E}-06$ & 3.19 & $0.25 \mathrm{E}-08$ & 4.10 & $0.48 \mathrm{E}-10$ & 4.59 \\
\hline
\end{tabular}

\begin{tabular}{|r|r|r|r|r|r|r|r|r|}
\hline & \multicolumn{2}{|c|}{$P^{1}$} & \multicolumn{2}{c|}{$P^{2}$} & \multicolumn{2}{c|}{$P^{3}$} & \multicolumn{2}{c|}{$P^{4}$} \\
\hline$N$ & $L^{\infty}$ error & order & $L^{\infty}$ error & order & $L^{\infty}$ error & order & $L^{\infty}$ error & order \\
\hline 10 & $0.29 \mathrm{E}+00$ & - & $0.24 \mathrm{E}-02$ & - & $0.69 \mathrm{E}-03$ & - & $0.13 \mathrm{E}-04$ & - \\
\hline 20 & $0.13 \mathrm{E}+00$ & 1.13 & $0.33 \mathrm{E}-03$ & 2.88 & $0.61 \mathrm{E}-04$ & 3.51 & $0.16 \mathrm{E}-05$ & 2.99 \\
\hline 40 & $0.58 \mathrm{E}-01$ & 1.15 & $0.37 \mathrm{E}-04$ & 3.15 & $0.58 \mathrm{E}-05$ & 3.39 & $0.13 \mathrm{E}-06$ & 3.64 \\
\hline 80 & $0.27 \mathrm{E}-01$ & 1.11 & $0.48 \mathrm{E}-05$ & 2.97 & $0.38 \mathrm{E}-06$ & 3.93 & $0.59 \mathrm{E}-08$ & 4.44 \\
\hline 160 & $0.13 \mathrm{E}-01$ & 1.07 & $0.59 \mathrm{E}-06$ & 3.00 & $0.23 \mathrm{E}-07$ & 4.07 & $0.25 \mathrm{E}-09$ & 4.57 \\
\hline
\end{tabular}

TABLE 4.2

Accuracy for $1 D$ non-convex, $H(u)=-\cos (u+1), t=0.5 / \pi^{2}$.

\begin{tabular}{|r|r|r|r|r|r|r|r|r|}
\hline & \multicolumn{2}{|c|}{$P^{1}$} & \multicolumn{2}{c|}{$P^{2}$} & \multicolumn{2}{c|}{$P^{3}$} & \multicolumn{2}{c|}{$P^{4}$} \\
\hline$N$ & $L^{1}$ error & order & $L^{1}$ error & order & $L^{1}$ error & order & $L^{1}$ error & order \\
\hline 10 & $0.84 \mathrm{E}-01$ & $\cdots$ & $0.10 \mathrm{E}-02$ & $-\cdots$ & $0.34 \mathrm{E}-03$ & - & $0.24 \mathrm{E}-04$ & - \\
\hline 20 & $0.36 \mathrm{E}-01$ & 1.23 & $0.15 \mathrm{E}-03$ & 2.75 & $0.30 \mathrm{E}-04$ & 3.49 & $0.13 \mathrm{E}-05$ & 4.28 \\
\hline 40 & $0.15 \mathrm{E}-01$ & 1.26 & $0.21 \mathrm{E}-04$ & 2.84 & $0.15 \mathrm{E}-05$ & 4.33 & $0.59 \mathrm{E}-07$ & 4.42 \\
\hline 80 & $0.68 \mathrm{E}-02$ & 1.14 & $0.27 \mathrm{E}-05$ & 2.97 & $0.94 \mathrm{E}-07$ & 4.00 & $0.21 \mathrm{E}-08$ & 4.78 \\
\hline
\end{tabular}

\begin{tabular}{|r|r|r|r|r|r|r|r|r|}
\hline & \multicolumn{2}{|c|}{$P^{1}$} & \multicolumn{2}{c|}{$P^{2}$} & \multicolumn{2}{c|}{$P^{3}$} & \multicolumn{2}{c|}{$P^{4}$} \\
\hline$N$ & $L^{\infty}$ error & order & $L^{\infty}$ error & order & $L^{\infty}$ error & order & $L^{\infty}$ error & order \\
\hline 10 & $0.18 \mathrm{E}+00$ & - & $0.15 \mathrm{E}-02$ & - & $0.11 \mathrm{E}-02$ & - & $0.99 \mathrm{E}-04$ & - \\
\hline 20 & $0.73 \mathrm{E}-01$ & 1.31 & $0.27 \mathrm{E}-03$ & 2.43 & $0.22 \mathrm{E}-03$ & 2.35 & $0.13 \mathrm{E}-04$ & 2.95 \\
\hline 40 & $0.31 \mathrm{E}-01$ & 1.24 & $0.47 \mathrm{E}-04$ & 2.54 & $0.18 \mathrm{E}-04$ & 3.63 & $0.59 \mathrm{E}-06$ & 4.44 \\
\hline 80 & $0.14 \mathrm{E}-01$ & 1.16 & $0.85 \mathrm{E}-05$ & 2.47 & $0.14 \mathrm{E}-05$ & 3.75 & $0.26 \mathrm{E}-07$ & 4.49 \\
\hline
\end{tabular}

The local Lax-Friedrichs flux (2.8) is used. At $t=0.5 / \pi^{2}$, the solution is still smooth. The accuracy of the numerical solution is listed in Table 4.2. We observe similar accuracy as in the previous example.

At $t=1.5 / \pi^{2}$, the solution has developed corner-like discontinuity in the derivative. The numerical result with 41 elements is shown in Fig. 4.2 .

Example 4.3. Riemann problem for the one dimensional equation with a non-convex flux:

$$
\left\{\begin{array}{l}
\phi_{t}+\frac{1}{4}\left(\phi_{x}^{2}-1\right)\left(\phi_{x}^{2}-4\right)=0, \quad-1<x<1 \\
\phi(x, 0)=-2|x|
\end{array} .\right.
$$

For this test problem, the discontinuous Galerkin method, as described in Sect. 2 and applied in the previous two examples, fails to "open up" the initial single discontinuity in the derivative sufficiently to 

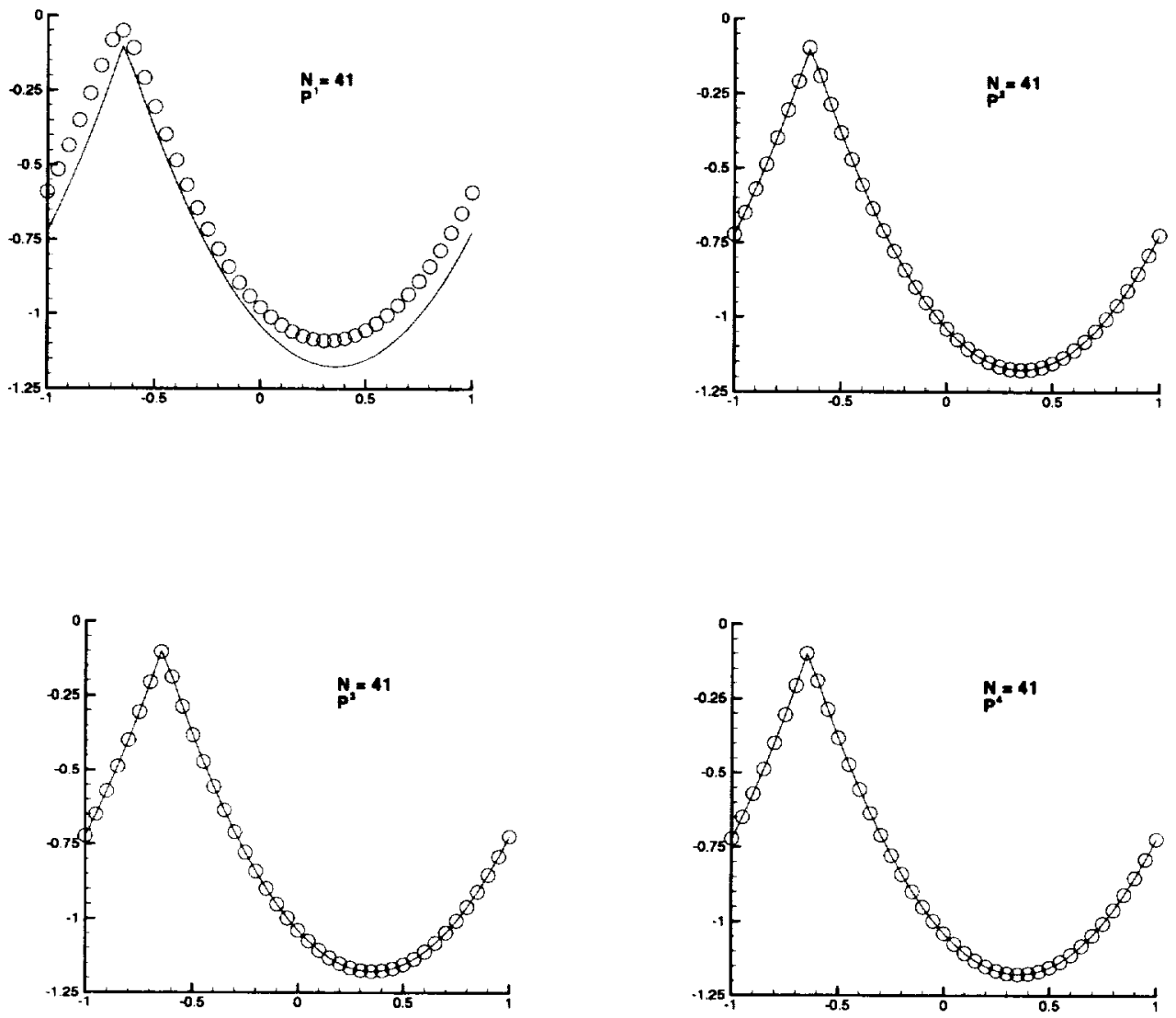

FIG. 4.1. One-dimensional Burgers' equation, $t=3.5 / \pi^{2}$.

generate the correct entropy solution. We have found out that a nonlinear total variation bounded limiting, described in detail in [6] for this one dimensional case, is needed for convergence towards the entropy solution. This and the 2D Riemann problem in Example 4.6 below are the only two examples in this paper in which we use the nonlinear limiting. We remark that for the finite difference schemes, such nonlinear limiting or the adaptive stencil in ENO is needed in most cases in order to enforce stability and to obtain non-oscillatory results.

Numerical results at $t=1$ with 81 elements, using the local Lax-Friedrichs flux (2.8), is shown in Fig. 4.3. The results of using the Godunov flux is shown in Fig. 4.4. We can see that while for $P^{1}$, the results of using two different monotone fluxes are significantly different in resolution, this difference is greatly reduced for higher order of accuracy. In most of the high order cases, the simple local Lax-Friedrichs flux (2.8) should be good enough.

Example 4.4. Two dimensional Burgers' equation:

$$
\left\{\begin{array}{l}
\phi_{t}+\frac{\left(\phi_{x}+\phi_{y}+1\right)^{2}}{2}=0, \quad-2<x<2,-2<y<2 \\
\phi(x, y, 0)=-\cos \left(\frac{\pi(x+y)}{2}\right)
\end{array}\right.
$$

with periodic boundary conditions. 

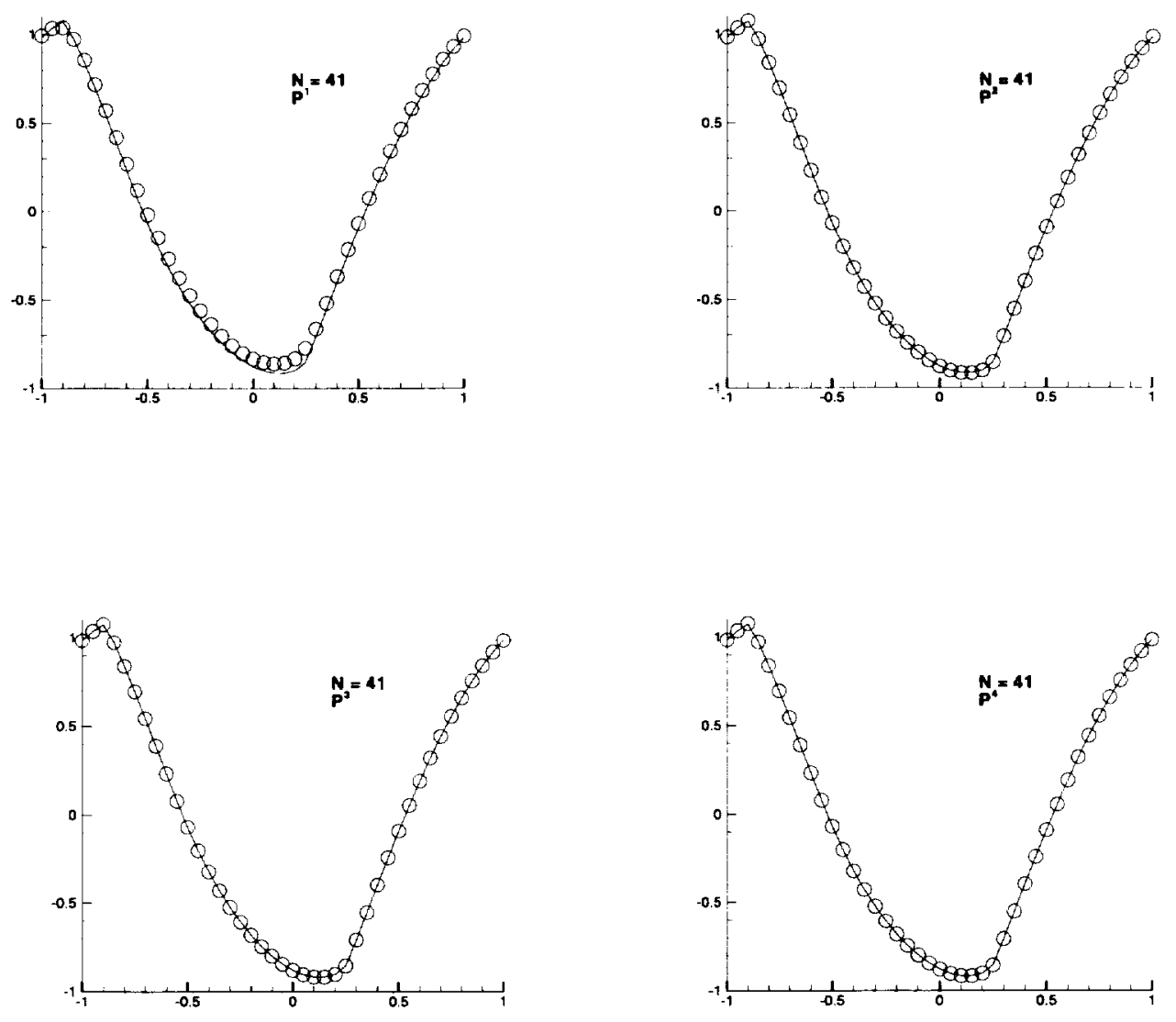

FIG. 4.2. One dimension non-convex, $H(u)=-\cos (u+1), t=1.5 / \pi^{2}$.

We first use uniform rectangular meshes and the local Lax-Friedrichs flux (3.8)-(3.9). At $t=0.5 / \pi^{2}$, the solution is still smooth. The errors and orders of accuracy are listed in Table 4.3. It seems that only $k$-th order of accuracy is achieved when $\varphi$ is a piecewise polynomial of degree $k$.

At $t=1.5 / \pi^{2}$, the solution has discontinuous derivatives. Fig. 4.5 is the graph of the numerical solution with $40 \times 40$ elements.

Next we use triangle based triangulation, the mesh with $h=\frac{1}{4}$ is shown in Fig. 4.6. The accuracy at $t=0.5 / \pi^{2}$ is shown in Table 4.4. Similar accuracy pattern is observed as in the rectangular case. The result at $t=1.5 / \pi^{2}$, when the derivative is discontinuous, is shown in Fig. 4.7.

Example 4.5. Two dimensional equation:

$$
\left\{\begin{array}{l}
\phi_{t}-\cos \left(\phi_{x}+\phi_{y}+1\right)=0, \quad-2<x<2,-2<y<2 \\
\phi(x, y, 0)=-\cos \left(\frac{\pi(x+y)}{2}\right)
\end{array}\right.
$$

with periodic boundary conditions.

For this example we use uniform rectangular meshes. The local Lax-Friedrichs flux (3.8)-(3.9) is used. The solution is smooth at $t=0.5 / \pi^{2}$. The accuracy of the numerical solution is shown in Table 4.5 .

The solution has developed a discontinuous derivative at $t=1.5 / \pi^{2}$. Results with $40 \times 40$ elements are shown in Fig. 4.8. 
TABLE 4.3

Accuracy for $2 D$ Burgers equation, rectangular mesh, $t=0.5 / \pi^{2}$.

\begin{tabular}{|r|r|r|r|r|r|r|}
\hline & \multicolumn{2}{|c|}{$P^{1}$} & \multicolumn{2}{c|}{$P^{2}$} & \multicolumn{2}{c|}{$P^{3}$} \\
\hline$N \times N$ & $L^{1}$ error & order & $L^{1}$ error & order & $L^{1}$ error & order \\
\hline $10 \times 10$ & $8.09 \mathrm{E}-02$ & - & $8.62 \mathrm{E}-03$ & - & $3.19 \mathrm{E}-03$ & - \\
\hline $20 \times 20$ & $3.36 \mathrm{E}-02$ & 1.268 & $1.72 \mathrm{E}-03$ & 2.325 & $3.49 \mathrm{E}-04$ & 3.192 \\
\hline $40 \times 40$ & $1.48 \mathrm{E}-02$ & 1.183 & $3.93 \mathrm{E}-04$ & 2.130 & $6.64 \mathrm{E}-05$ & 2.394 \\
\hline $80 \times 80$ & $6.88 \mathrm{E}-03$ & 1.105 & $9.74 \mathrm{E}-05$ & 2.013 & $1.14 \mathrm{E}-05$ & 2.542 \\
\hline $160 \times 160$ & $3.31 \mathrm{E}-03$ & 1.056 & $2.45 \mathrm{E}-05$ & 1.991 & $1.68 \mathrm{E}-06$ & 2.763 \\
\hline
\end{tabular}

\begin{tabular}{|r|r|r|r|r|r|r|}
\hline & \multicolumn{2}{|c|}{$P^{1}$} & \multicolumn{2}{c|}{$P^{2}$} & \multicolumn{2}{c|}{$P^{3}$} \\
\hline$N \times N$ & $L^{\infty}$ error & order & $L^{\infty}$ error & order & $L^{\infty}$ error & order \\
\hline $10 \times 10$ & $2.62 \mathrm{E}-01$ & - & $3.56 \mathrm{E}-02$ & - & $8.65 \mathrm{E}-03$ & - \\
\hline $20 \times 20$ & $1.14 \mathrm{E}-01$ & 1.201 & $8.40 \mathrm{E}-03$ & 2.083 & $1.16 \mathrm{E}-03$ & 2.899 \\
\hline $40 \times 40$ & $5.00 \mathrm{E}-02$ & 1.189 & $2.02 \mathrm{E}-03$ & 2.056 & $1.98 \mathrm{E}-04$ & 2.551 \\
\hline $80 \times 80$ & $2.39 \mathrm{E}-02$ & 1.065 & $4.92 \mathrm{E}-04$ & 2.038 & $3.13 \mathrm{E}-05$ & 2.661 \\
\hline $160 \times 160$ & $1.16 \mathrm{E}-02$ & 1.043 & $1.21 \mathrm{E}-04$ & 2.024 & $4.41 \mathrm{E}-06$ & 2.827 \\
\hline
\end{tabular}

TABLE 4.4

Accuracy for $2 D$ Burgers equation, triangular mesh, $t=0.5 / \pi^{2}$.

\begin{tabular}{|r|r|r|r|r|r|r|r|r|}
\hline & \multicolumn{4}{|c|}{$P^{2}$} & \multicolumn{5}{c|}{$P^{3}$} \\
\hline$h$ & $L^{1}$ error & order & $L^{\infty}$ error & order & $L^{1}$ error & order & $L^{\infty}$ error & order \\
\hline 1 & $5.48 \mathrm{E}-02$ & - & $1.52 \mathrm{E}-01$ & -- & $1.17 \mathrm{E}-02$ & - & $2.25 \mathrm{E}-02$ & - \\
\hline $1 / 2$ & $1.35 \mathrm{E}-02$ & 2.02 & $6.26 \mathrm{E}-02$ & 1.28 & $1.35 \mathrm{E}-03$ & 3.12 & $4.12 \mathrm{E}-03$ & 2.45 \\
\hline $1 / 4$ & $2.94 \mathrm{E}-03$ & 2.20 & $1.55 \mathrm{E}-02$ & 2.01 & $1.45 \mathrm{E}-04$ & 3.22 & $4.31 \mathrm{E}-04$ & 3.26 \\
\hline $1 / 8$ & $6.68 \mathrm{E}-04$ & 2.14 & $3.44 \mathrm{E}-03$ & 2.17 & $1.71 \mathrm{E}-05$ & 3.08 & $7.53 \mathrm{E}-05$ & 2.52 \\
\hline
\end{tabular}

TABLE 4.5

Accuracy, $2 D, H(u, v)=-\cos (u+v+1), t=0.5 / \pi^{2}$.

\begin{tabular}{|c|c|c|c|c|c|c|}
\hline & \multicolumn{2}{|c|}{$P^{1}$} & \multicolumn{2}{|c|}{$P^{2}$} & \multicolumn{2}{|c|}{$P^{3}$} \\
\hline$N \times N$ & $L^{1}$ error & order & $L^{1}$ error & order & $L^{1}$ error & order \\
\hline $10 \times 10$ & $6.47 \mathrm{E}-02$ & - & 8.31E-03 & - & $1.35 \mathrm{E}-02$ & - \\
\hline $20 \times 20$ & $2.54 \mathrm{E}-02$ & 1.349 & $1.93 \mathrm{E}-03$ & 2.106 & $1.57 \mathrm{E}-03$ & 3.104 \\
\hline $40 \times 40$ & $1.05 \mathrm{E}-02$ & 1.274 & $4.58 \mathrm{E}-04$ & 2.075 & 2.39E-04 & 2.716 \\
\hline $80 \times 80$ & $4.74 \mathrm{E}-03$ & 1.147 & $1.13 \mathrm{E}-04$ & 2.019 & $2.89 \mathrm{E}-05$ & 3.048 \\
\hline \multirow[t]{2}{*}{$160 \times 160$} & $2.23 \mathrm{E}-03$ & 1.088 & $2.83 \mathrm{E}-05$ & 1.997 & $4.38 \mathrm{E}-06$ & 2.722 \\
\hline & \multicolumn{2}{|l|}{$P^{1}$} & \multicolumn{2}{|l|}{$P^{2}$} & \multicolumn{2}{|c|}{$P^{3}$} \\
\hline$N \times N$ & $L^{\infty}$ error & order & $L^{\infty}$ error & order & $L^{\infty}$ error & order \\
\hline $10 \times 10$ & $1.47 \mathrm{E}-01$ & - & $1.88 \mathrm{E}-02$ & & $2.36 \mathrm{E}-02$ & $\cdots$ \\
\hline $20 \times 20$ & $6.75 \mathrm{E}-02$ & 1.123 & $7.34 \mathrm{E}-03$ & 1.357 & $3.44 \mathrm{E}-03$ & 2.778 \\
\hline $40 \times 40$ & $2.65 \mathrm{E}-02$ & 1.349 & $1.83 \mathrm{E}-03$ & 2.004 & $4.59 \mathrm{E}-04$ & 2.906 \\
\hline $80 \times 80$ & $1.18 \mathrm{E}-02$ & 1.167 & $4.55 \mathrm{E}-04$ & 2.008 & $5.78 \mathrm{E}-05$ & 2.989 \\
\hline $160 \times 160$ & $2.23 \mathrm{E}-03$ & 1.088 & $1.13 \mathrm{E}-04$ & 2.010 & $8.54 \mathrm{E}-06$ & 2.759 \\
\hline
\end{tabular}



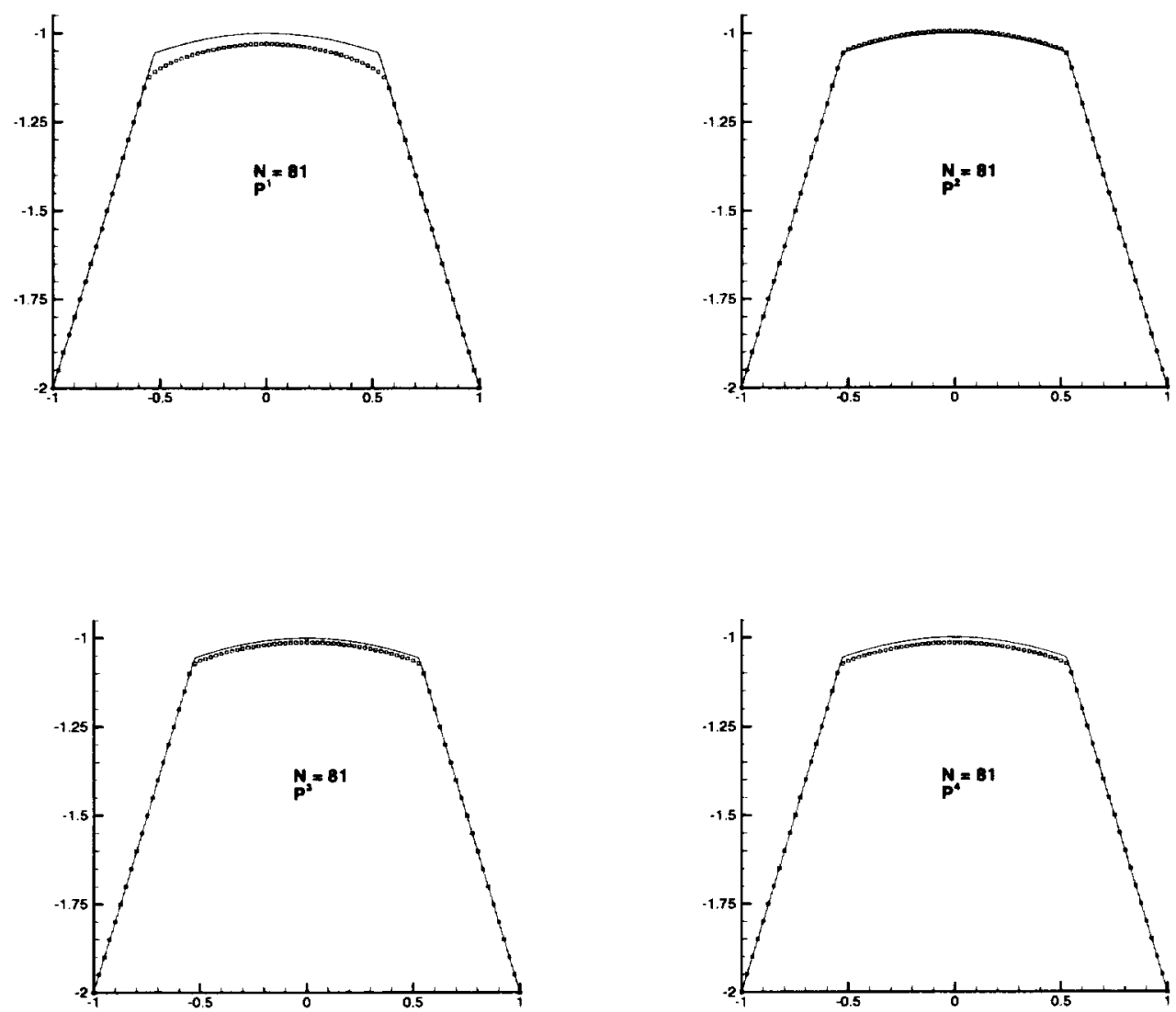

FIG. 4.3. One dimension Riemann problem, local Lax-Friedrichs flux, $H(u)=\frac{1}{4}\left(u^{2}-1\right)\left(u^{2}-4\right), t=1$.

Example 4.6. Two dimensional Riemann problem:

$$
\left\{\begin{array}{l}
\phi_{t}+\sin \left(\phi_{x}+\phi_{y}\right)=0, \quad-1<x<1,-1<y<1 \\
\phi(x, y, 0)=\pi(|y|-|x|)
\end{array}\right.
$$

For this example we use a uniform rectangular mesh with $40 \times 40$ elements. The local Lax-Friedrichs flux (3.8)-(3.9) is used. As it was mentioned in Example 4.3, we have found out that a nonlinear limiting $[8],[9]$ is needed, for convergence towards an entropy solution. We show the numerical solution at $t=1$ in Fig. 4.9 .

Example 4.7. The problem of a propagating surface:

$$
\left\{\begin{array}{l}
\phi_{t}-(1-\epsilon K) \sqrt{1+\phi_{x}^{2}+\phi_{y}^{2}}=0, \quad 0<x<1,0<y<1 \\
\phi(x, y, 0)=1-\frac{1}{4}(\cos (2 \pi x-1))(\cos (2 \pi y-1))
\end{array}\right.
$$

where $K$ is the mean curvature defined by

$$
K=-\frac{\phi_{x x}\left(1+\phi_{y}^{2}\right)-2 \phi_{x y} \phi_{x} \phi_{y}+\phi_{y y}\left(1+\phi_{x}^{2}\right)}{\left(1+\phi_{x}^{2}+\phi_{y}^{2}\right)^{\frac{3}{2}}},
$$

and $\varepsilon$ is a small constant. Periodic boundary condition is used. 

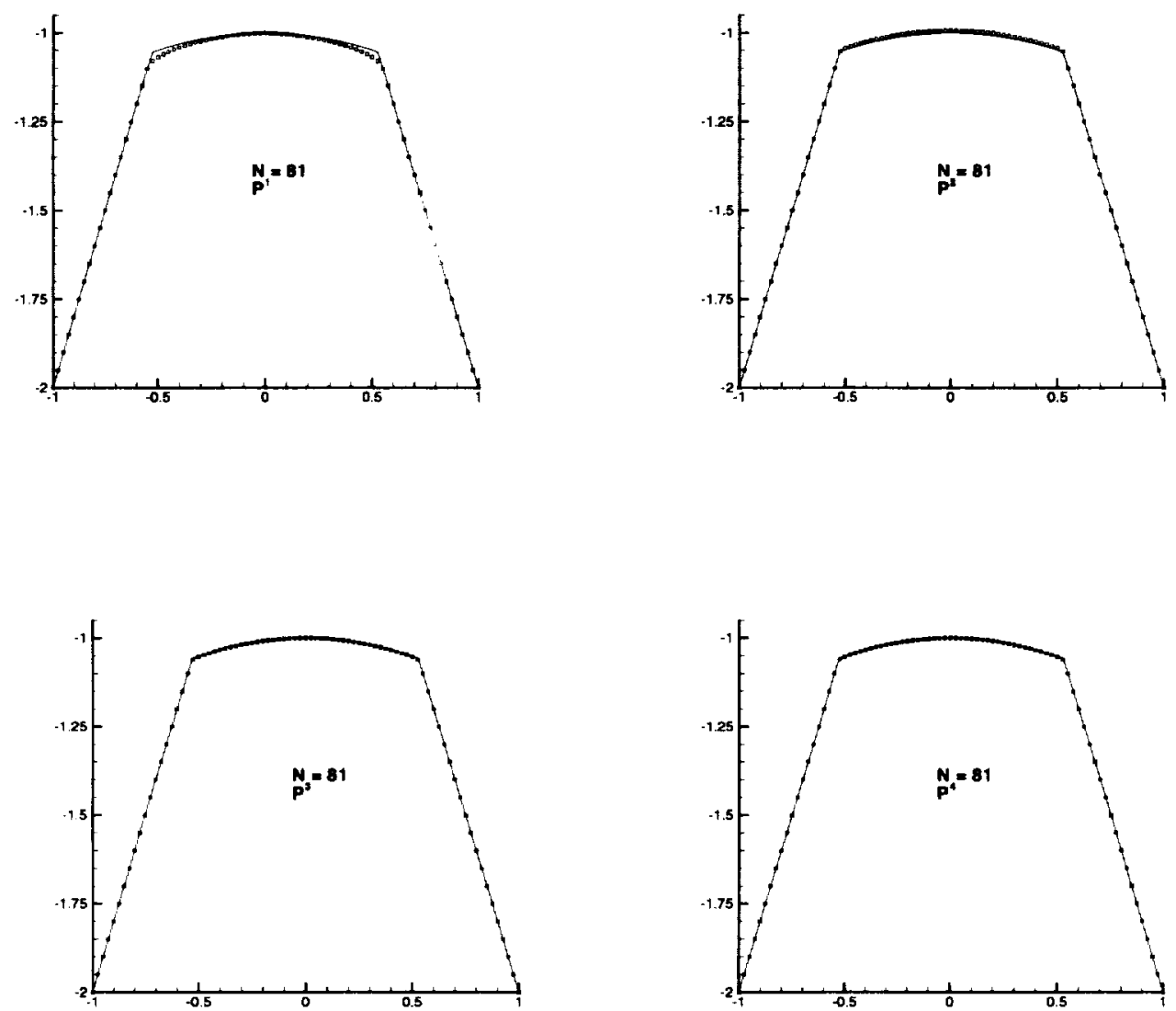

FIG. 4.4. One dimension Riemann problem, Godunov flux, $H(u)=\frac{1}{4}\left(u^{2}-1\right)\left(u^{2}-4\right), t=1$.

This problem was studied in [22] by using the finite difference ENO schemes. We apply the discontinuous Galerkin method, with the second derivative terms handled by the local discontinuous Galerkin techniques presented and analyzed in [10], which amounts to solving the following system

$$
\left\{\begin{array}{l}
u_{t}-\left(\sqrt{1+u^{2}+v^{2}}+\varepsilon \frac{p\left(1+v^{2}\right)-2 q u v+r\left(1+u^{2}\right)}{1+u^{2}+v^{2}}\right)_{x}=0 \\
v_{t}-\left(\sqrt{1+u^{2}+v^{2}}+\varepsilon \frac{p\left(1+v^{2}\right)-2 q u v+r\left(1+u^{2}\right)}{1+u^{2}+v^{2}}\right)_{y}=0 \\
p-u_{x}=0 \\
q-u_{y}=0 \\
r-v_{y}=0
\end{array}\right.
$$

using the discontinuous Galerkin method. The details of the method, especially the choices of fluxes, which are important for stability, can be found in [10].

We first use a uniform rectangular mesh of $50 \times 50$ elements and the local Lax-Friedrichs flux (3.8)-(3.9). The results of $\varepsilon=0$ (pure convection) and $\varepsilon=0.1$ are presented in Fig. 4.10 and Fig. 4.11, respectively. Notice that the surface at $t=0$ is shifted downward by 0.35 in order to show the detail of the solution at $t=0.3$.

Next we use a triangulation shown in Fig. 4.12. We refine the mesh around the center of domain where the solution develops discontinuous derivatives (for the $\varepsilon=0$ case). There are 2146 triangles and 1108 nodes 

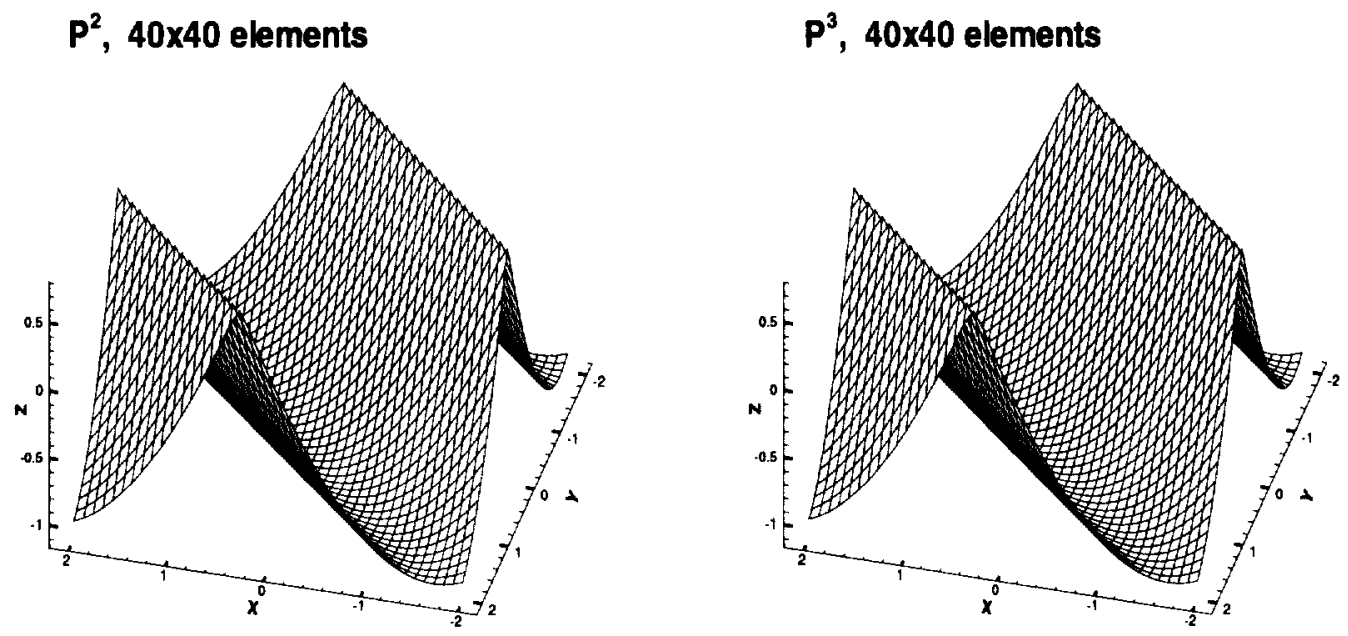

FIG. 4.5. Two dimension Burgers' equation, rectangular mesh, $t=1.5 / \pi^{2}$.

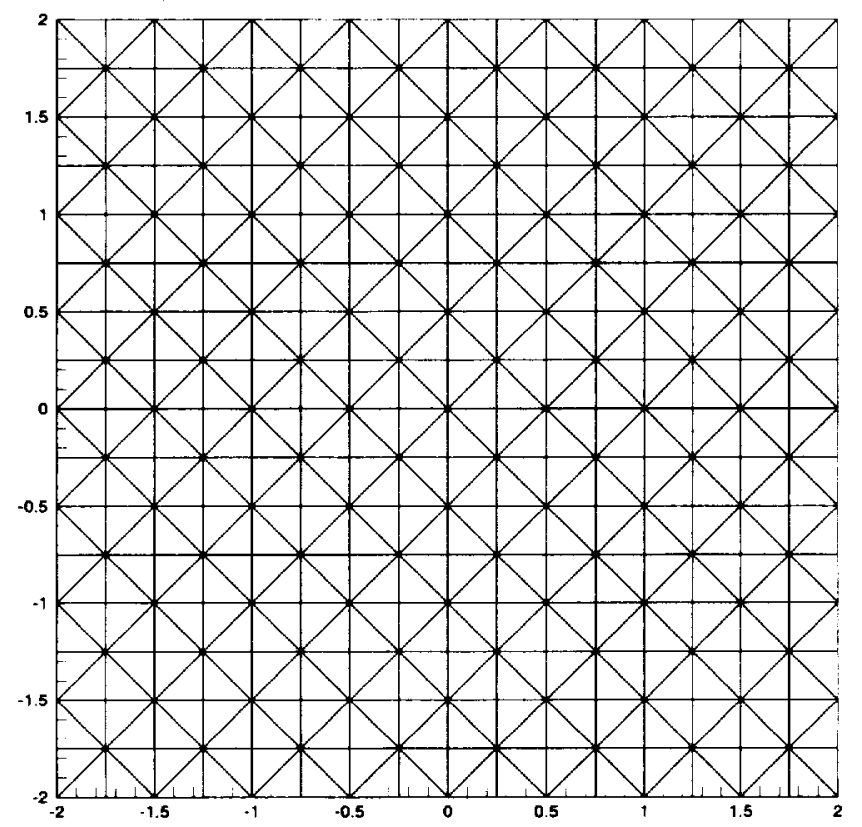

FIG. 4.6. Triangulation for two dimensional Burgers equation, $h=\frac{1}{4}$.

in this triangulation. The solutions are displayed in Fig. 4.13 and Fig. 4.14, respectively, for $\varepsilon=0$ (pure convection) and $\varepsilon=0.1$. Notice that we again shift the solution at $t=0.0$ downward by 0.35 to show the detail of the solutions at later time.

Example 4.8. The problem of a propagating surface on a unit disk. The equation is the same as (4.7) in 


$$
P^{2}, h=1 / 8
$$

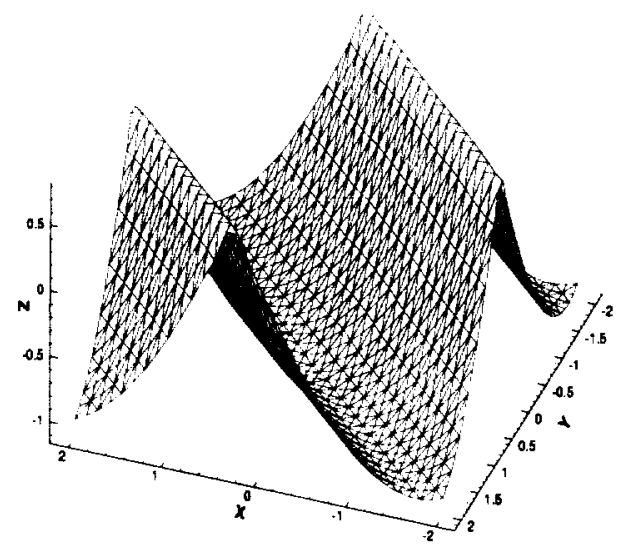

$P^{3}, h=1 / 8$

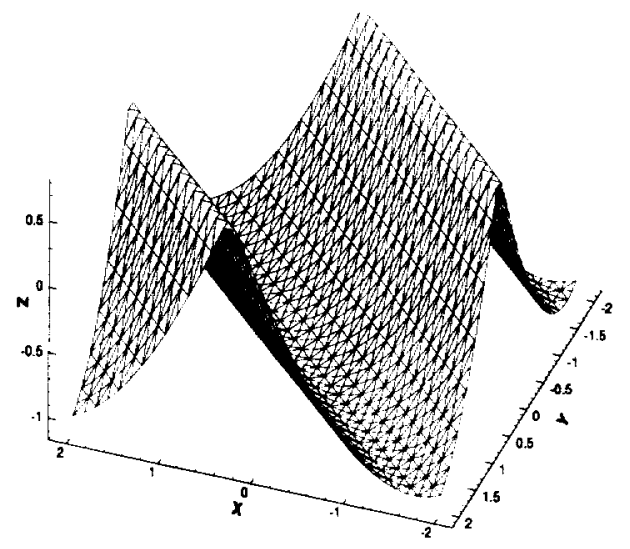

FIG. 4.7. Two dimension Burgers' equation, triangular mesh, $t=1.5 / \pi^{2}$.
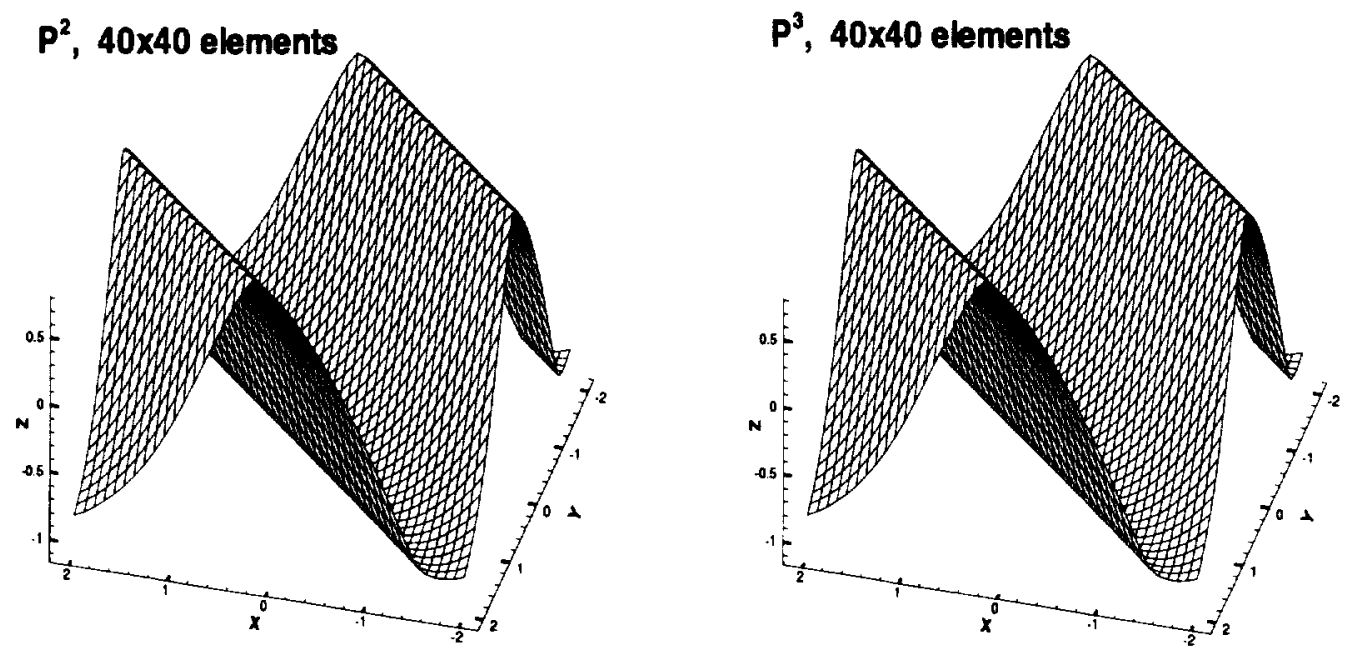

Fig. 4.8. Two dimensional, $H(u, v)=-\cos (u+v+1), t=1.5 / \pi^{2}$.

the previous example, but it is solved on a unit disk $x^{2}+y^{2}<1$ with an initial condition

$$
\phi(x, y, 0)=\sin \left(\frac{\pi\left(x^{2}+y^{2}\right)}{2}\right)
$$

and a Neumann type boundary condition $\nabla \phi=0$.

It is difficult to use rectangular meshes for this problem. Instead we use the triangulation shown in Fig. 4.15. Notice that we have again refined the mesh near the center of the domain where the solution develops discontinuous derivatives. There are 1792 triangles and 922 nodes in this triangulation. The solutions with $\varepsilon=0$ are displayed in Fig. 4.16. Notice that the solution at $t=0$ is shifted downward by 0.2 to show the detail of the solution at later time. 

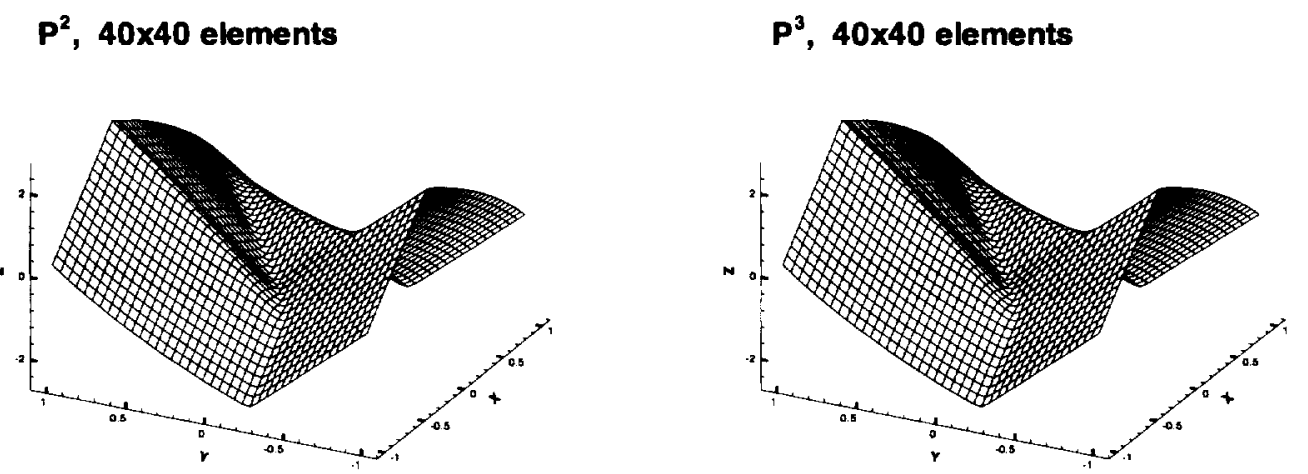

FIG. 4.9. Two dimensional Riemann problem, $H(u, v)=\sin (u+v), t=1$.
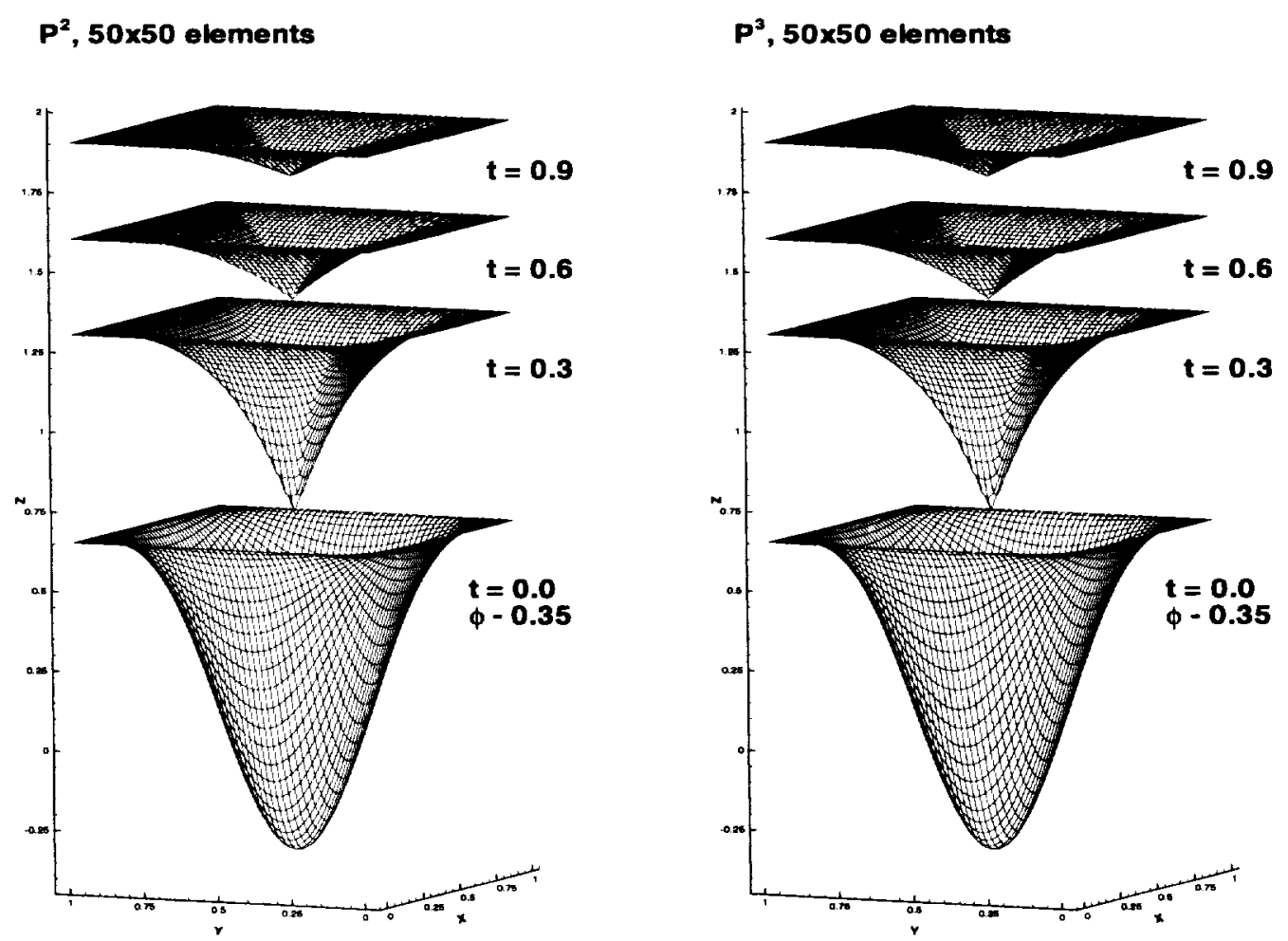

FIG. 4.10. Propagating surfaces, rectangular mesh, $\epsilon=0$.

The solution with $\varepsilon=0.1$ are displayed in Fig. 4.17. Notice that the solution at $t=0$ is again shifted downward by 0.2 to show the detail of the solution at later time. 

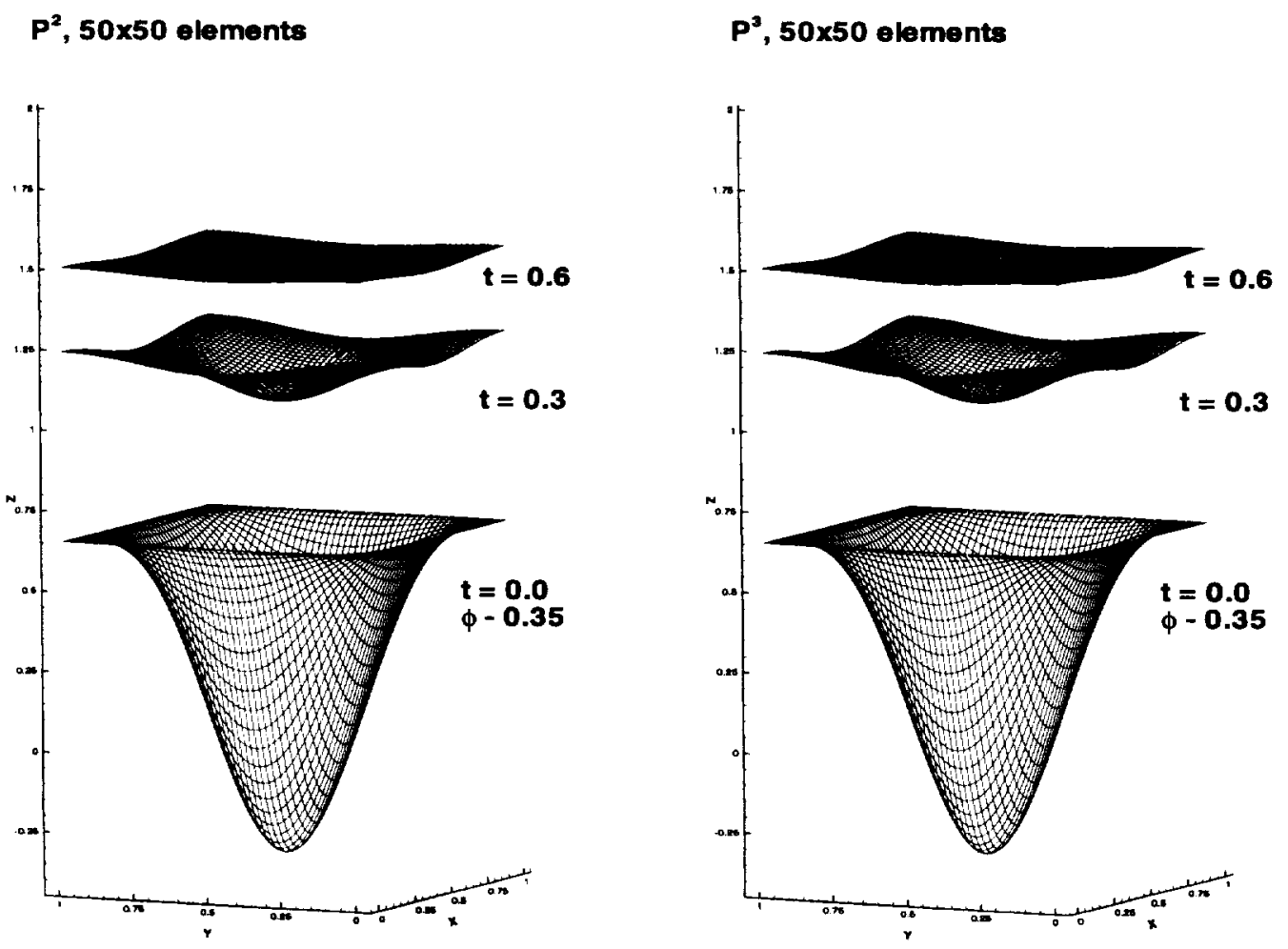

FIG. 4.11. Propagating surfaces, rectangular mesh, $\varepsilon=0.1$.

Example 4.9. A problem from optimal control [23]:

(4.10) $\left\{\begin{array}{l}\phi_{t}+(\sin y) \phi_{x}+\left(\sin x+\operatorname{sign}\left(\phi_{y}\right)\right) \phi_{y}-\frac{1}{2} \sin ^{2} y-(1-\cos x)=0,-\pi<x<\pi,-\pi<y<\pi \\ \phi(x, y, 0)=0\end{array}\right.$

with periodic boundary conditions. We use a uniform rectangular mesh of $40 \times 40$ elements and the local Lax-Friedrichs flux (3.8)-(3.9). The solution at $t=1$ is shown in Fig. 4.18, while the optimal control $w=\operatorname{sign}\left(\phi_{y}\right)$ is shown in Fig. 4.19.

Notice that our method computes $\nabla \phi$ as an independent variable. It is very desirable for those problems in which the most interesting features are contained in the first derivatives of $\phi$, as in this optimal control problem.

Example 4.10. A problem from computer vision [24]:

$$
\left\{\begin{array}{l}
\phi_{t}+I(x, y) \sqrt{1+\phi_{x}^{2}+\phi_{y}^{2}}-1=0, \quad-1<x<1,-1<y<1 \\
\phi(x, y, 0)=0
\end{array}\right.
$$

with $\phi=0$ as the boundary condition. The steady state solution of this problem is the shape lighted by a source located at infinity with vertical direction. The solution is not unique if there are points at which $I(x, y)=1$. Conditions must be prescribed at those points where $I(x, y)=1$. Since our method is a finite 


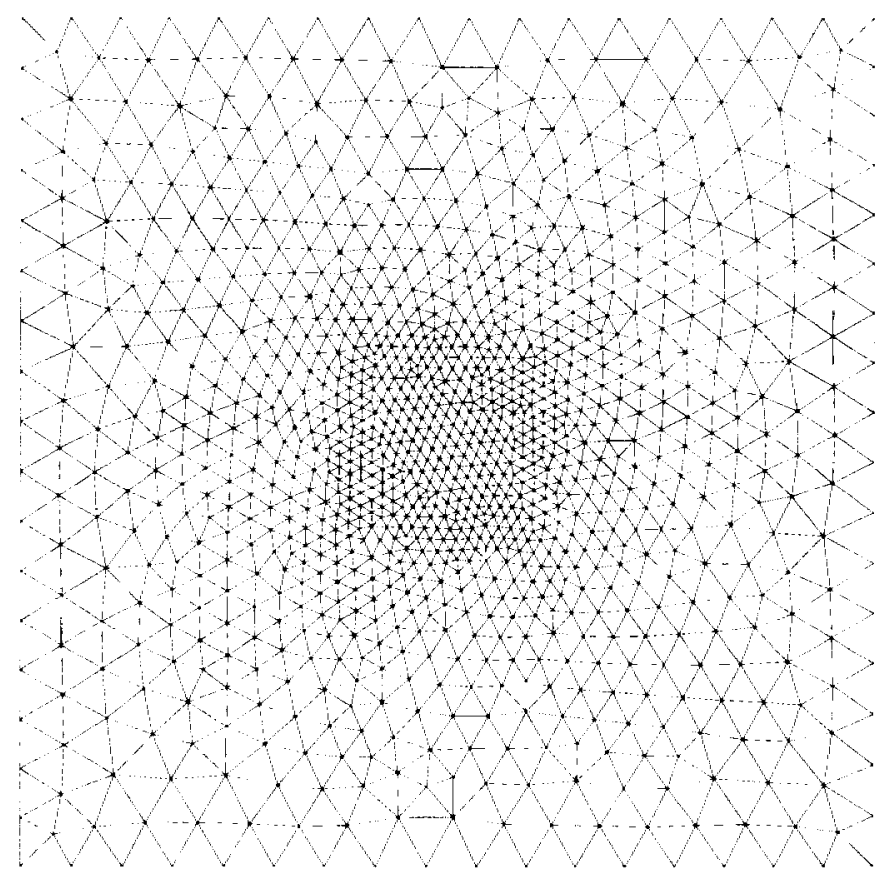

FiG. 4.12. Triangulation used for the propagating surfaces.

element method, we need to prescribe suitable conditions at the correspondent elements. We take

$$
I(x, y)=1 / \sqrt{1+(1-|x|)^{2}+(1-|y|)^{2}}
$$

The exact steady solution is $\phi(x, y, \infty)=(1-|x|)(1-|y|)$. We use a uniform rectangular mesh of $40 \times 40$ elements and the local Lax-Friedrichs flux (3.8)-(3.9). We impose the exact boundary conditions for $u=$ $\phi_{x}, v=\phi_{y}$ from the above exact steady solution, and take the exact value at one point (the lower left corner) to recover $\phi$. The results for $P^{2}$ and $P^{3}$ are presented in Fig. 4.20, while Fig. 4.21 contains the history of iterations to the steady state.

Next we take

$$
I(x, y)=1 / \sqrt{1+4 y^{2}\left(1-x^{2}\right)^{2}+4 x^{2}\left(1-y^{2}\right)^{2}}
$$

The exact steady solution is $\phi(x, y, \infty)=\left(1-x^{2}\right)\left(1-y^{2}\right)$. We again use a uniform rectangular mesh of $40 \times 40$ elements, the local Lax-Friedrichs flux (3.8)-(3.9), impose the exact boundary conditions for $u=\phi_{x}, v=\phi_{y}$ from the above exact steady solution, and take the exact value at one point (the lower left corner) to recover $\phi$. A continuation method is used, with the steady solution using

$$
I_{\varepsilon}(x, y)=1 / \sqrt{1+4 y^{2}\left(1-x^{2}\right)^{2}+4 x^{2}\left(1-y^{2}\right)^{2}+\varepsilon}
$$

for bigger $\varepsilon$ as the initial condition for smaller $\varepsilon$. The sequence of $\varepsilon$ used are $\varepsilon=0.2,0.05,0$. The results for $P^{2}$ and $P^{3}$ are presented in Fig. 4.22 .

5. Concluding Remarks.. We have developed and tested a class of discontinuous Galerkin methods for solving nonlinear Hamilton-Jacobi equations. These methods have the advantage of easy handling of complicated geometry and local mesh refinements, as well as efficient parallel implementations. Numerical examples in one and two space dimensions are shown to illustrate the capability of the methods. 


$$
\mathbf{P}^{2} \text {, triangles }
$$

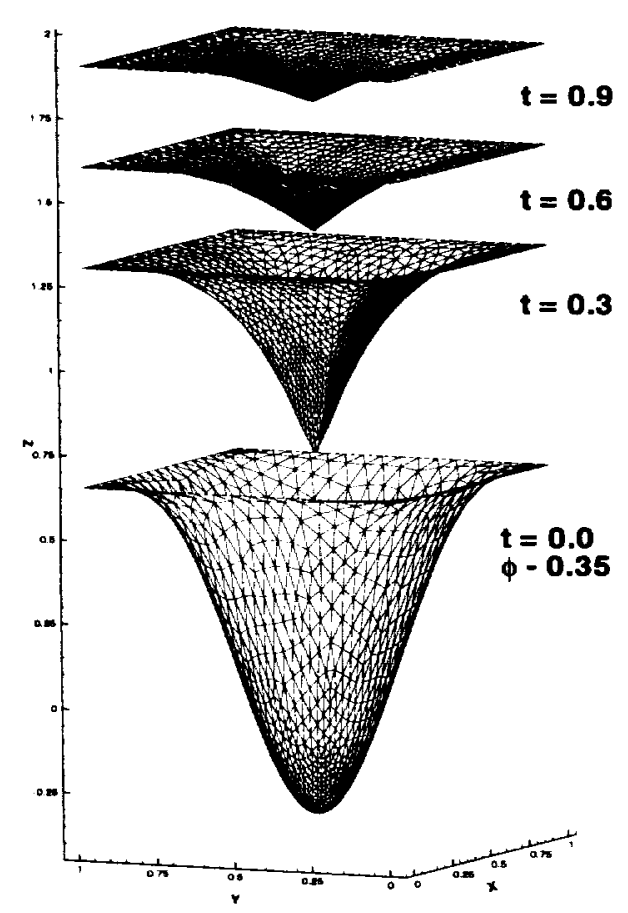

$\mathbf{P}^{3}$, triangles

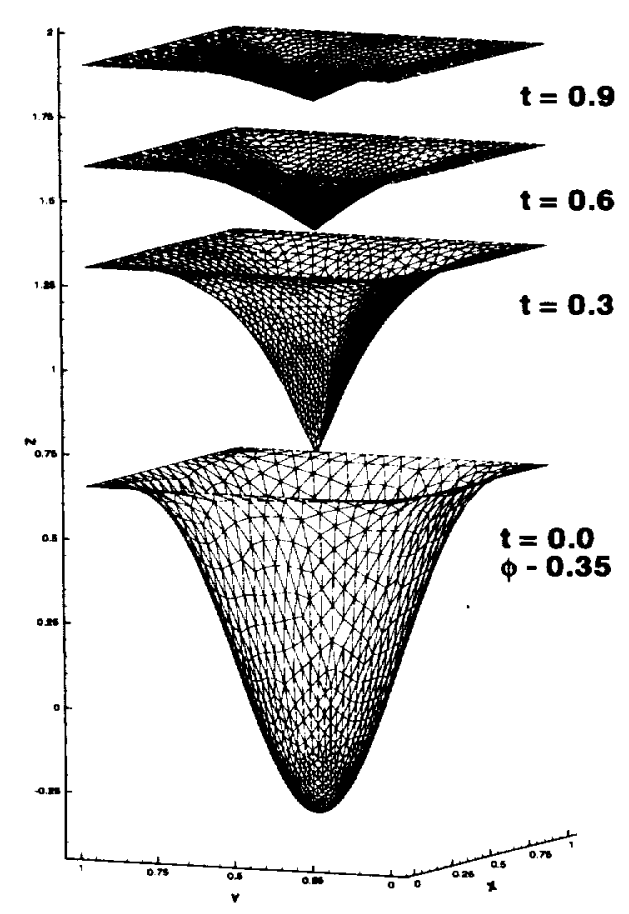

FIG. 4.13. Propagating surfaces, triangular mesh, $\varepsilon=0$.

\section{REFERENCES}

[1] R. ABGRALL, On essentially non-oscillatory schemes on unstructured meshes: analysis and implementation, J. Comput. Phys., vol. 114 (1994), pp.45-58.

[2] R. Abgrall, Numerical discretization of the first-order Hamilton-Jacobi equation on triangular meshes., Comm. Pure Appl. Math., vol. 49 (1996), pp.1339-1373.

[3] R. Biswas, K.D. Devine And J. Flaherty, Parallel, adaptive finite element methods for conservation laws, Appl. Numer. Math., vol. 14 (1994), pp.255-283.

[4] B. Cockburn, An introduction to the discontinuous Galerkin method for convection-dominated problems, in Advanced Numerical Approximation of Nonlinear Hyperbolic Equations, A. Quarteroni, Editor, Lecture Notes in Mathematics, CIME subseries, Springer-Verlag, to appear.

[5] B. CockbURn AND C.-W. ShU, The Runge-Kutta local projection $P^{1}$-discontinuous Galerkin method for scalar conservation laws, $M^{2} A N$, vol. 25 (1991), pp.337-361.

[6] B. Cockburn AND C.-W. SHU, TVB Runge-Kutta local projection discontinuous Galerkin finite element method for scalar conservation laws II: general framework, Math. Comp., vol. 52 (1989), pp.411-435.

[7] B. COCKBURN, S.-Y. LIN AND C.-W. SHU, TVB Runge-Kutta local projection discontinuous Galerkin finite element method for conservation laws III: one dimensional systems, J. Comput. Phys., vol. 84 (1989), pp.90-113. 

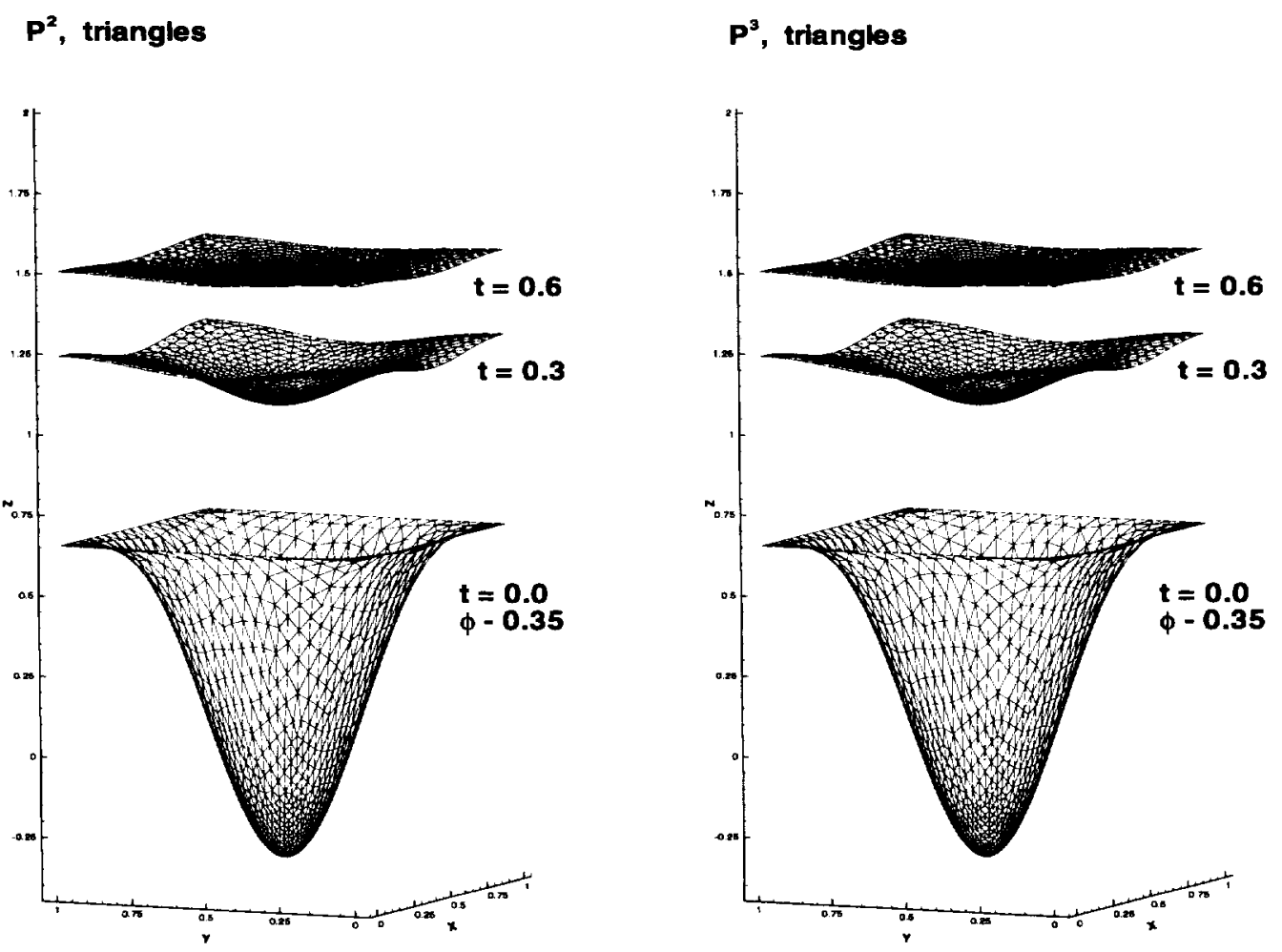

FIG. 4.14. Propagating surfaces, triangular mesh, $\varepsilon=0.1$.

[8] B. Cockburn, S. Hou, AND C.-W. Shu, TVB Runge-Kutta local projection discontinuous Galerkin finite element method for conservation laws IV: the multidimensional case, Math. Comp., vol. 54 (1990), pp.545-581.

[9] B. Cockburn and C.-W. Shu, TVB Runge-Kutta local projection discontinuous Galerkin finite element method for scalar conservation laws V: multidimensional systems, J. Comput. Phys., to appear.

[10] B. COCKBURN AND C.-W. SHU, The local discontinuous Galerkin method for time-dependent convection diffusion systems, SIAM J. Numer. Anal., to appear.

[11] M. Crandall and P.L. Lions, Viscosity solutions of Hamilton-Jacobi equations, Trans. Amer. Math. Soc., vol. 277 (1983), pp.1-42.

[12] M. Crandall And P.L. Lions, Two approximations of solutions of Hamilton-Jacobi equations, Math. Comp., vol. 43 (1984), pp.1-19.

[13] S. Gottlieb AND C.-W. Shu, Total variation diminishing Runge-Kutta schemes, Math. Comp., to appear.

[14] A. Harten, B. Engquist, S. Osher and S. Chakravarthy, Uniformly high order essentially nonoscillatory schemes, III, J. Comput. Phys., vol. 71 (1987), pp.231-303.

[15] G. JiANG AND D. PENG, Weighted ENO schemes for Hamilton-Jacobi equations, SIAM J. Sci. Comput., submitted.

[16] G. Jiang AND C.-W. Shu, On cell entropy inequality for discontinuous Galerkin methods, Math. Comp., vol. 62 (1994), pp.531-538. 


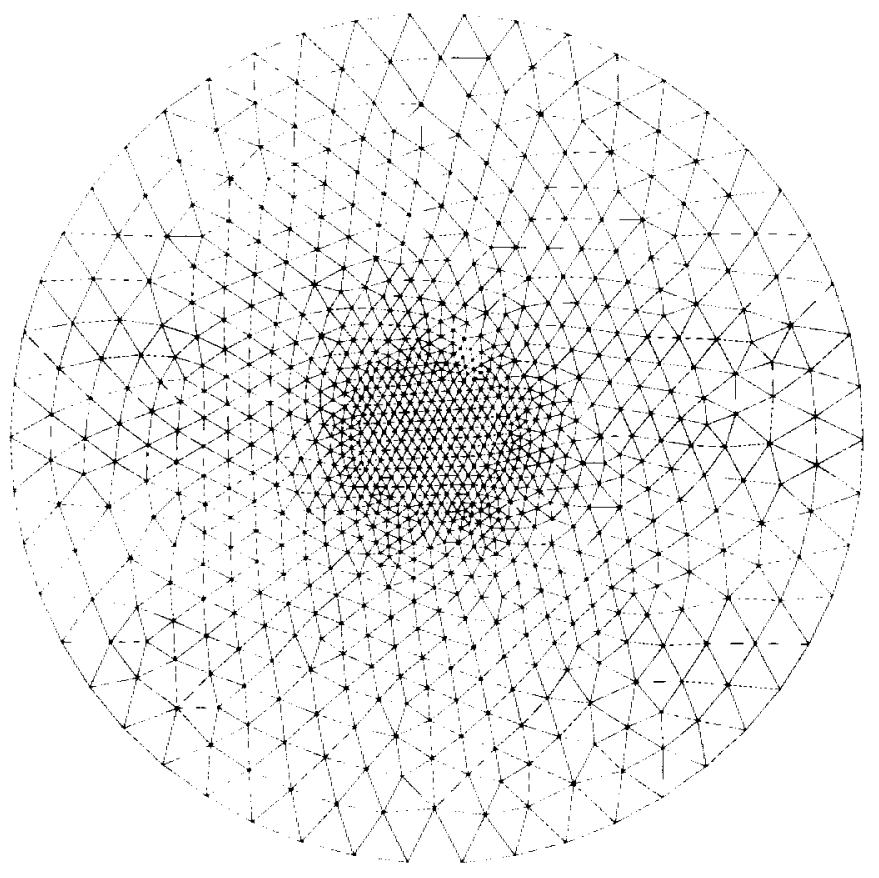

FIG. 4.15. Triangulation for the propagating surfaces on a disk.

[17] G. Jiang AND C.-W. ShU, Efficient implementation of weighted ENO schemes, J. Comput. Phys., vol. 126 (1996), pp.202-228.

[18] S. JIN AND Z. XIN, Numerical passage from systems of conservation laws to Hamilton-Jacobi equations, SIAM J. Numer. Anal., submitted.

[19] F. LAFON AND S. OSHER, High order two dimensional nonoscillatory methods for solving HamiltonJacobi scalar equations, J. Comput. Phys., vol. 123 (1996), pp.235-253.

[20] X.-D. LiU, S. Osher And T. Chan, Weighted essentially nonoscillatory schemes, J. Comput. Phys., vol. 115 (1994), pp.200 212.

[21] I. LOMTEV AND G. KARNIADAKIS, A discontinuous spectral/hp element Galerkin method for the NavierStokes equations on unstructured grids, preprint. CFM Report No. 97-10, 1997, Brown University.

[22] S. Osher AND J. SEThIAN, Fronts propagating with curvature dependent speed: algorithms based on Hamilton-Jacobi formulations, J. Comput. Phys., vol. 79 (1988), pp.12-49.

[23] S. Osher AND C.-W. SHU, High-order essentially nonoscillatory schemes for Hamilton-Jacobi equations, SIAM J. Numer. Anal., vol. 28 (1991), pp.907-922.

[24] E. RouY AND A. Tourin, A viscosity solutions approach to shape-from- shading, SIAM J. Numer. Anal., vol. 29 (1992), pp.867-884.

[25] C.-W. SHU AND S. OSHER, Efficient implementation of essentially non-oscillatory shock capturing schemes, J. Comput. Phys., vol. 77 (1988), pp.439-471.

[26] C.-W. SHU AND S. OSHER, Efficient implementation of essentially non-oscillatory shock capturing schemes II, J. Comput. Phys., vol. 83 (1989), pp.32-78. 

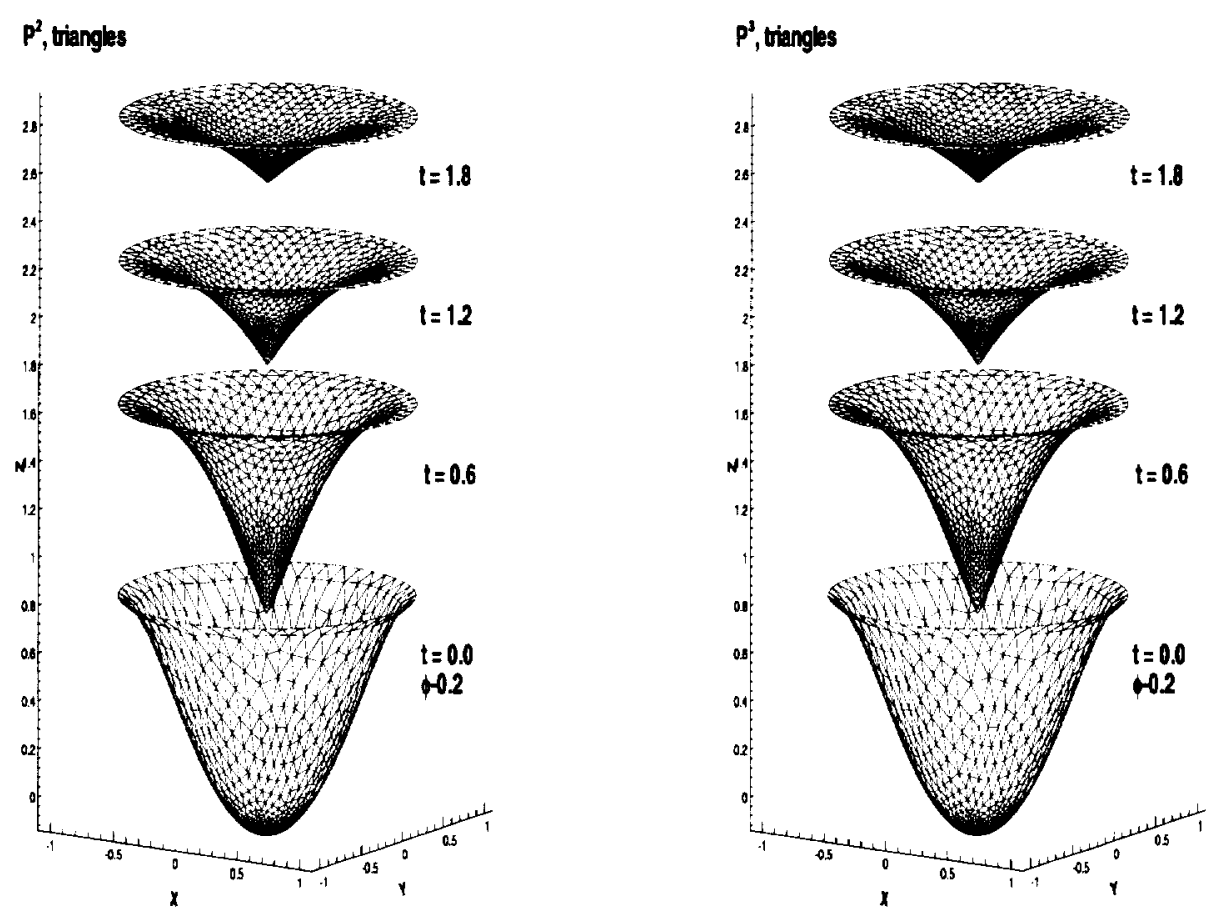

FIG. 4.16. Propagating surfaces on a disk, triangular mesh, $\varepsilon=0$.
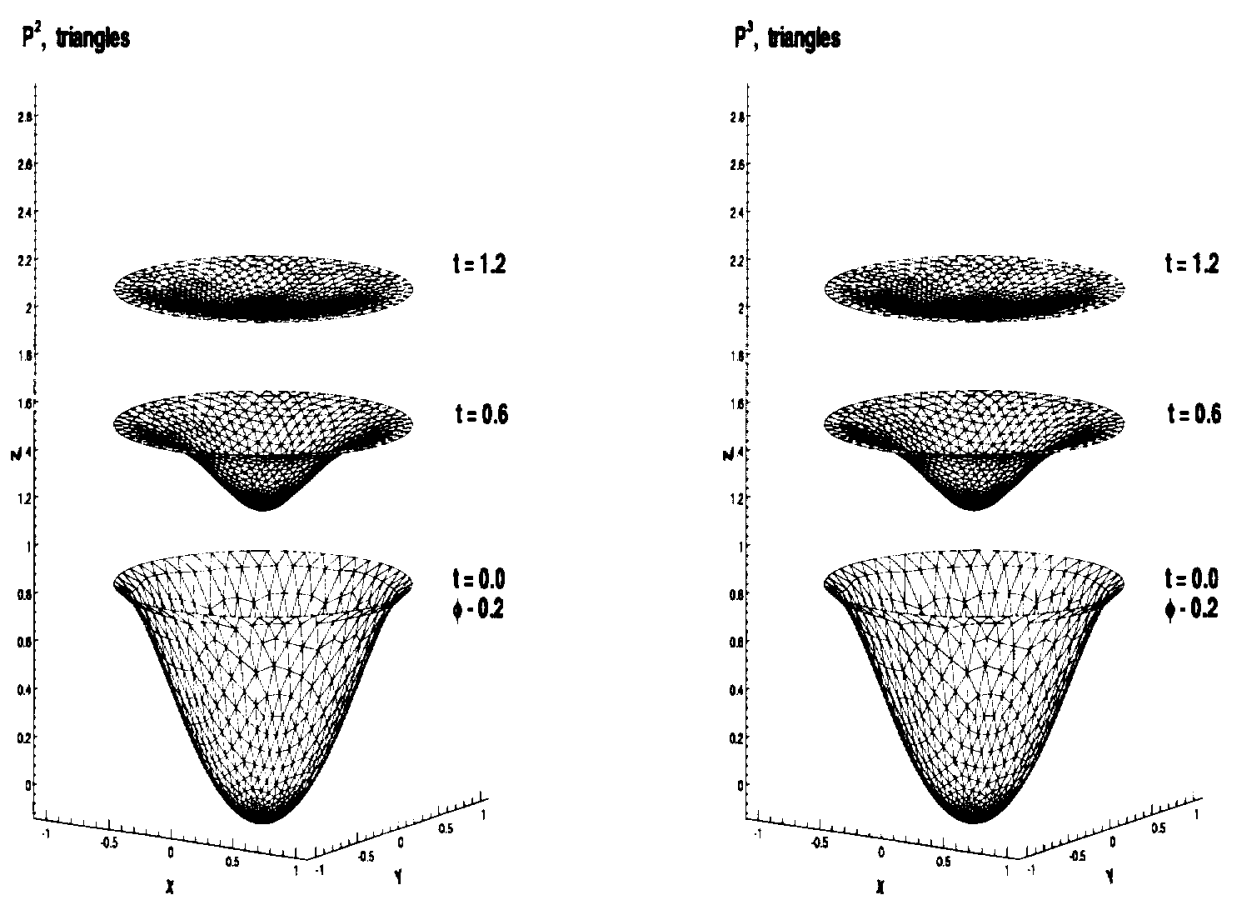

FIG. 4.17. Propagating surfaces on a disk, triangular mesh, $\varepsilon=0.1$. 
$P^{2}, 40 \times 40$ elements

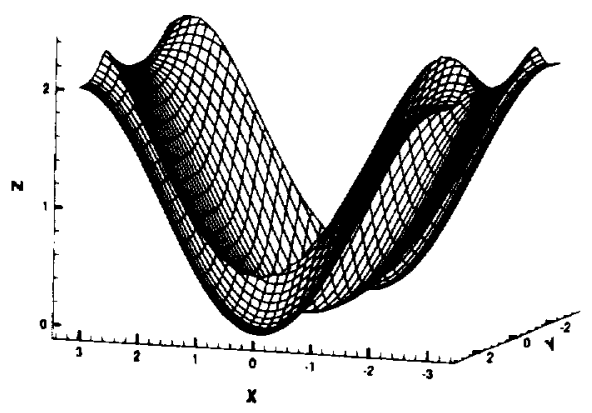

$P^{3}, 40 \times 40$ elements

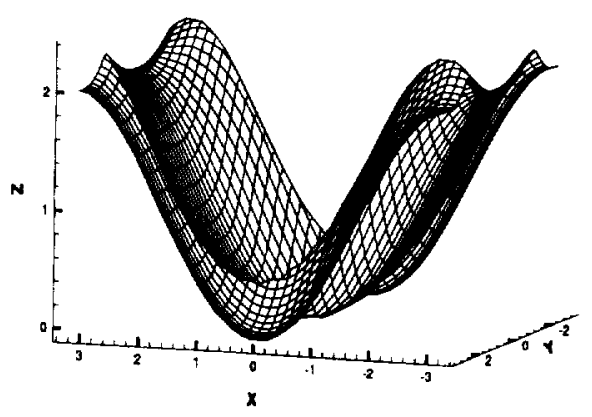

FIG. 4.18. Control problem, $t=1$.

$P^{2}, 40 \times 40$ elements

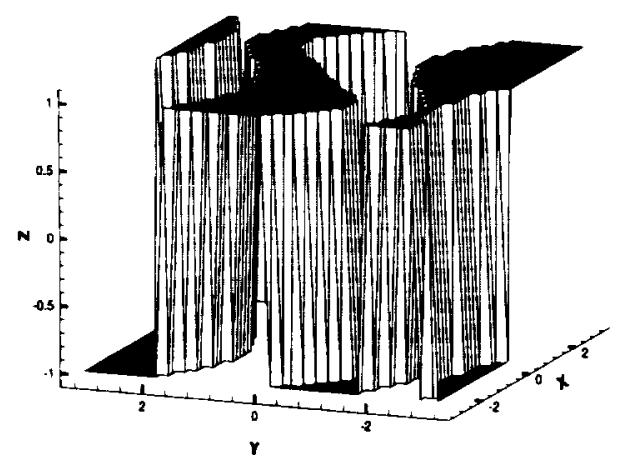

$P^{3}, 40 \times 40$ elements

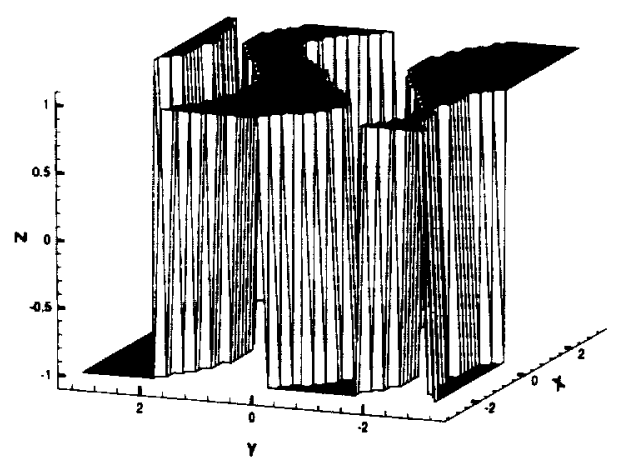

FIG. 4.19. Control problem, $t=1, w=\operatorname{sign}\left(\phi_{y}\right)$. 

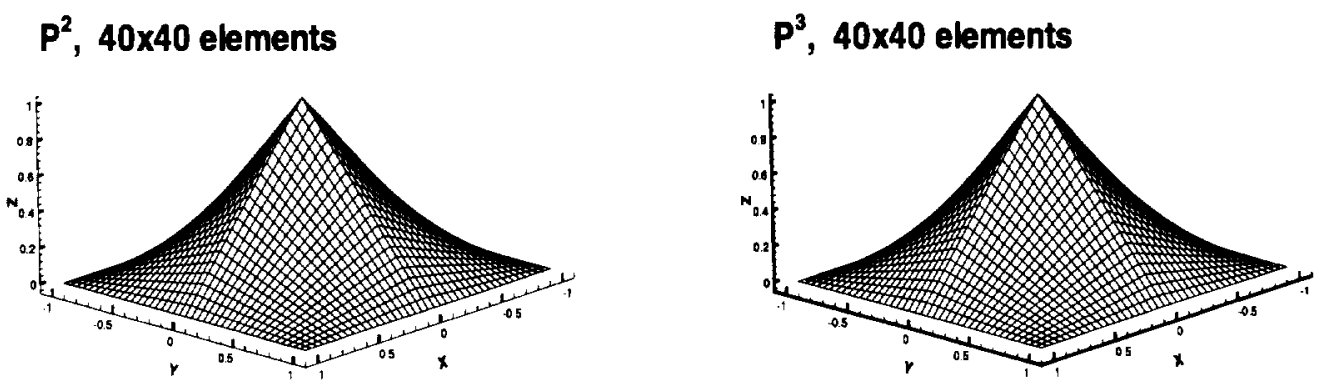

FiG. 4.20. Computer vision problem, $\phi(x, y, \infty)=(1-|x|)(1-|y|)$.
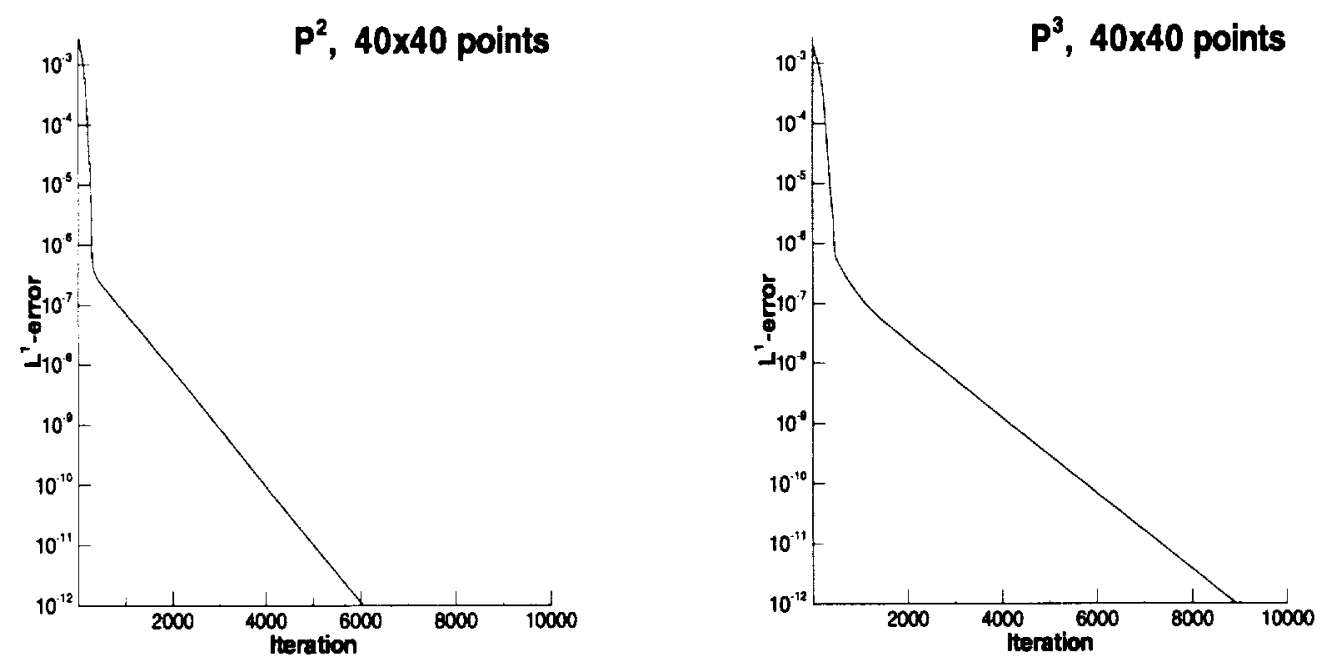

FIG. 4.21. Computer vision problem, history of iterations. 

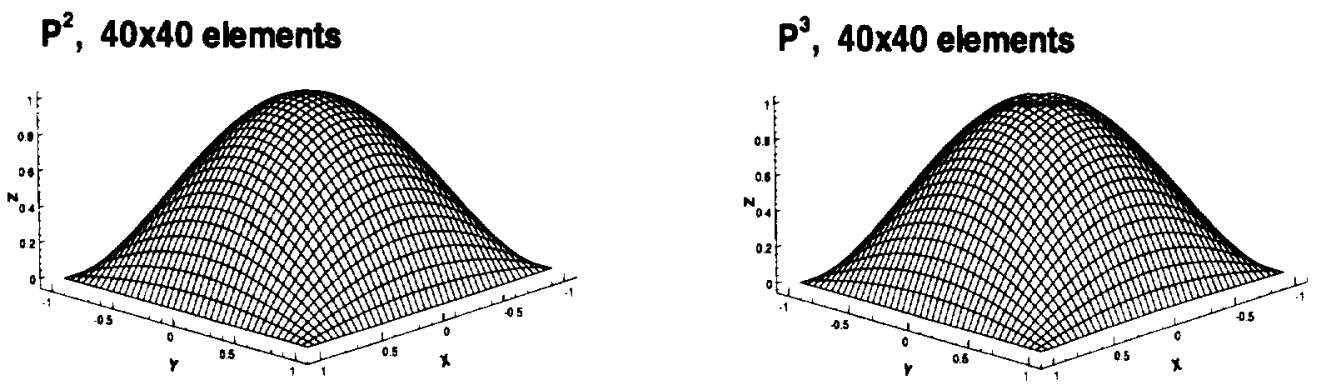

FIG. 4.22. Computer vision problem, $\phi(x, y, \infty)=\left(1-x^{2}\right)\left(1-y^{2}\right)$. 
Public reporting burden for this collection of information is estimated to average 1 hour per response, including the time for reviewing instructions, searching existing data sources, gathering and maintaining the data needed, and completing and reviewing the collection of information. Send comments regarding this burden estimate or any other aspect of this Davis Highway, Suite 1204, Arlington, VA 22202-4302, and to the Office of Management and Budget. Paperwork Reduction Project (0704-0188), Washington, DC 20503.

1. AGENCY USE ONLY(Leave blank) $\begin{gathered}\text { 2. REPORT DATE } \\ \text { January } 1998\end{gathered} \quad \begin{gathered}\text { 3. REPORT TYPE AND DATES COVERED } \\ \text { Contractor Report }\end{gathered}$

4. TITLE AND SUBTITLE

A Discontinuous Galerkin Finite Element Method for HamiltonJacobi Equations

C NAS1-19480

WU 505-90-52-01

6. AUTHOR(S)

Changqing $\mathrm{Hu}$

Chi-Wang Shu

7. PERFORMING ORGANIZATION NAME(S) AND ADDRESS(ES)

Institute for Computer Applications in Science and Engineering

Mail Stop 403, NASA Langley Research Center

Hampton, VA 23681-0001

8. PERFORMING ORGANIZATION REPORT NUMBER

ICASE Report No. 98-2

9. SPONSORING/MONITORING AGENCY NAME(S) AND ADDRESS(ES)

National Aeronautics and Space Administration

Langley Research Center

Hampton, VA 23681-2199

10. SPONSORING/MONITORING

AGENCY REPORT NUMBER

NASA/CR-1998-206903

ICASE Report No. 98-2

\section{SUPPLEMENTARY NOTES}

Langley Technical Monitor: Dennis M. Bushnell

Final Report

Submitted to Journal of Computational Physics

\begin{tabular}{l|l} 
12a. DISTRIBUTION/AVAILABILITY STATEMENT & 12b. DISTRIBUTION CODE \\
Unclassified-Unlimited & \\
Subject Category 64 & \\
Distribution: Nonstandard & \\
Availability: NASA-CASI (301)621-0390 &
\end{tabular}

13. ABSTRACT (Maximum 200 mords)

In this paper, we present a discontinuous Galerkin finite element method for solving the nonlinear Hamilton-Jacobi equations. This method is based on the Runge-Kutta discontinuous Galerkin finite element method for solving conservation laws. The method has the flexibility of treating complicated geometry by using arbitrary triangulation, can achieve high order accuracy with a local, compact stencil, and are suited for efficient parallel implementation. One and two dimensional numerical examples are given to illustrate the capability of the method.

\begin{tabular}{|c|c|c|c|}
\hline \multirow{2}{*}{\multicolumn{3}{|c|}{$\begin{array}{l}\text { 14. SUBJECT TERMS } \\
\text { Hamilton-Jacobi equations, discontinuous Galerkin, high order accuracy }\end{array}$}} & \multirow{2}{*}{$\begin{array}{c}\text { 15. NUMBER OF PAGES } \\
29 \\
\text { 16. PRICE CODE } \\
\mathrm{A} 03 \\
\end{array}$} \\
\hline & & & \\
\hline $\begin{array}{l}\text { 17. SECURITY CLASSIFICATION } \\
\text { OF REPORT } \\
\text { Unclassified }\end{array}$ & $\begin{array}{l}\text { 18. SECURITY CLASSIFICATION } \\
\text { OF THIS PAGE } \\
\text { Unclassified }\end{array}$ & $\begin{array}{l}\text { 19. SECURITY CLASSIFICATION } \\
\text { OF ABSTRACT }\end{array}$ & $\begin{array}{l}\text { 20. LIMITATION } \\
\text { OF ABSTRACT }\end{array}$ \\
\hline
\end{tabular}


